\title{
Overcoming Chemoresistance to Vemurafenib in Melanoma via Targeted Inhibition of PCK1 Using 3- mercaptopropionic Acid
}

\section{Ming Ren}

Zhong Shan Hospital: Zhongshan Hospital Fudan University

\section{Lu Wang}

Zhongshan Hospital Fudan University

\section{Xin-Yi Deng}

Zhongshan Hospital Fudan University

\section{Zi-Xu Gao}

Zhongshan Hospital Fudan University

\section{Qiang Wang}

Zhongshan Hospital Fudan University

\section{Meng-Yuan Tao}

Zhongshan Hospital Fudan University

\section{Kang-Jie Shen}

Zhongshan Hospital Fudan University

\section{Yi-Teng Ding}

Zhongshan Hospital Fudan University

\section{Le-Qi Qian}

Zhongshan Hospital Fudan University

\section{Chuan-Yuan Wei}

Zhongshan Hospital Fudan University

Jian-Ying Gu ( $\sigma$ zhongshanhospital@163.com )

Zhong Shan Hospital: Zhongshan Hospital Fudan University

\section{Research}

Keywords: BRAF inhibitor, resistance, PCK1, melanoma,3-mercaptopropionic acid

Posted Date: May 7th, 2021

DOI: https://doi.org/10.21203/rs.3.rs-462600/v1 
License: (c) (i) This work is licensed under a Creative Commons Attribution 4.0 International License. Read Full License 


\section{Abstract}

Background:BRAF inhibitors are the mainstay treatment for melanoma with the V600E mutation, but its resistance to BRAFi remains a clinical challenge. Therefore, it is necessary to explore the mechanism of BRAFi resistance and develop new therapeutic targets.

Methods:We established an A2058 melanoma cell line with acquired resistance to vemurafenib in vitro. RNA sequencing was used to identify the target gene and signaling pathway which were related to the resistance.A series of in vitro assays were applied to confirm the function of PCK1, including test of scratch,transwell,cell viability,flow cytometry,sphere formation,western blot and ROS detection.Western blot was taken to identify the activation of PI3K/Akt pathway.We constructed the subcutaneous xenograft model of melanoma,and the mice were randomly injected with DMSO,vemurafenib,and combination of vemurafenib and 3-mercaptopropionic acid.Finally, tumor size,ROS in tissue, and immunohistochemistry were analyzed to validate the findings.

Results:We identified that the activation of PI3K/Akt pathway led to the overexpression of phosphoenolpyruvate carboxykinase 1, a key enzyme of gluconeogenesis. An elevated PCK1 level induced intracellular metabolic reprogramming, thereby lowering oxidative stress contributed to the chemoresistance to vemurafenib. 3-mercaptopropionic acid, an antihyperglycemic agent, could inhibit the viability of PCK1 then bring oxidative damage to drug-resistant cells. As a result, 3-mercaptopropionic acid sensitized the cells to the killing effect of vemurafenib, exerting a synergistic anti-tumor effect in combination with vemurafenib.

Conclusions:Our study demonstrates that the PI3K/Akt-PCK1-ROS axis plays an important role in BRAFiresistant melanoma and that using the antihyperglycemic agent 3-MPA is a feasible strategy to restore its therapeutic sensitivity.

\section{Background}

The incidence of melanoma, a malignant tumor of melanocytes that arises in the skin, has been increasing in recent years. ${ }^{1}$ BRAF mutation is a prominent feature of melanoma, with studies pointing to the presence of serine/threonine protein kinase B-Raf activating mutation in $50 \sim 60 \%$ of patients with advanced melanoma, and with the V600E mutation being the most common, comprising $74-86 \%$ of all BRAF mutations. ${ }^{2,3}$ This oncogenic mutation activates the downstream kinase MEK/ERK within the MAPK pathway, promoting melanoma progression. ${ }^{4}$ Inhibitors designed to target the BRAF mutation, such as vemurafenib and dabrafenib, have achieved pioneering results. The median PFS of vemurafenib was found to be higher than that of dabrafenib (5.8 months versus 7.3 months). ${ }^{5,6}$ However, patients can only benefit from the use of these inhibitors in the early stages of treatment, since inevitable acquired resistance is the major factor contributing to treatment failure. Studies show that 'paradoxical activation' includes mutations in NRAS or MEK1/MEK2, and a high expression level of COT, EGFR, PDGFR, IGF1R, or MET, which has encouraged clinicians to investigate a combination of inhibitors that effectively block 
the MAPK signaling pathway. ${ }^{7,8}$ Based on the 'paradoxical activation' of the MAPK pathway via BRAF inhibition, the BRAF-MEK inhibitor combination was further investigated in phase III clinical trial COMBI-d. The results showed that patients could benefit more from combination therapy than from vemurafenib alone (median PFS, 11.4 months; OS, 25.1 months versus 7.3 months, 18.7 months). ${ }^{9}$ Therefore, "Darabfenib + Trametinib" combination therapy is recommended as the main chemotherapy for advanced melanoma patients. Innovative strategies are still urgently needed to improve the precision and persistence of melanoma therapies.

The process of metabolic remodeling is crucial for melanoma cells to adapt to the tumor microenvironment (TME) and maintain growth and metastasis. ${ }^{10}$ Phosphoenolpyruvate carboxykinase 1 (PCK1, PEPCK-C), a cytoplasmic form of PEPCK, is a rate limiting enzyme, which decarboxylate in the second step of gluconeogenesis after the carboxylation of pyruvate, and then phosphorylates oxaloacetic acid to form phosphoenolpyruvate (PEP). ${ }^{11,12}$ As a central molecule regulating glycolysis, the tricarboxylic acid cycle (TCA), and gluconeogenesis, PCK1 exerts diametrically opposite biological effects in different tissues. ${ }^{13}$ In gluconeogenic tissues, such as the liver and kidneys, the overexpression of PCK1 acts as a suppressor ${ }^{14}$ because it exacerbates gluconeogenesis and hinders both glycolysis and the TCA cycle, leading to nutrient stress and energy homeostasis. ${ }^{15}$ Interestingly, in the colon or lungs, where glycogen is not produced, the overexpression of PCK1 abundantly generates NADPH to reduce the levels of reactive oxygen species (ROS) through the pentose phosphate pathway (PPP). ${ }^{16}$ Thus, a high level of PCK1 promotes cancer exacerbation when carcinogenesis occurs in intestinal and lung cancers. However, the mechanism of PCK1 during melanoma progression and chemoresistance remains unknown and regulation of metabolic reprogramming may be an Achilles' heel of cancer, with important implications.

In this study, A2058, a cell line harboring the BRAF V600E mutation, was used to construct a vemurafenibresistant cell line in vitro. Using next-generation sequencing, we found that hyperactivation of the Akt pathway and its downstream molecule PCK1 was associated with resistance to BRAF inhibitors (BRAFi) in melanoma cells. PCK1, a key enzyme of PPP, promotes resistance in non-gluconeogenic tissues to oxidative stress by synthesizing a large amount of NADPH to maintain oxidation homeostasis, which keeps melanoma cells alive and proliferating under drug pressure. Based on this, we revealed the role of PCK1 inhibitor 3-mercaptopropionic acid (3-MPA) in reversing the metabolic reprogramming of melanoma cells using in vivo and in vitro experiments. We found that 3-MPA can significantly enhance the therapeutic effect of BRAFi and is therefore a novel strategy for the clinical treatment of melanoma.

\section{Methods}

\section{Establishment of BRAFi-resistant melanoma cells}

A2058 cells were inoculated in a culture flask, and a high dose of vemurafenib(PLX4032, Cat\#HY-L032) (MedChemExpress, USA) was used for treatment. When most of the cells had died, the dead cells were washed away and the medium was changed to a drug-free medium. Post reaching confluence, the cells 
were digested and re-inoculated into a new culture flask, and some cells were cryopreserved for RNA-seq. The cells were treated with high density vemurafenib until the cells reached $30 \%$ confluence. This process was repeated 3 times, and we obtained R0, R1, R2, and R3 cells, which were then used for RNA-seq analysis. Melanoma cells in the exponential growth phase were inoculated into culture bottles. After $48 \mathrm{~h}$, the cells were transferred to a drug-free medium and expanded until the next mitotic phase. Then, the above steps were repeated using vemurafenib that was two times more concentrated. At the same time, cell death was observed every day, the fresh complete medium containing vemurafenib was replaced, and CCK8 analysis was performed regularly until melanoma cells grew stably in the medium. This process lasted for six months, and transcriptome sequencing was performed once every two months; thus, 4 samples (R0, R1, R2, and R3) were obtained.

\section{Cell proliferation and drug treatments}

A cell proliferation experiment was carried out on a 96 well plate. The cells in the exponential growth phase were analyzed using the CCK8(Beyotime,China) method. The inoculation density used was $4 \times 10^{3}$ cells/well. The treatment started at $24 \mathrm{~h}$ post inoculation and lasted for $72 \mathrm{~h}$. Then, $10 \mu \mathrm{l}$ of a CCK8 solution was added to each well, and the cells were cultured at $37^{\circ} \mathrm{C}$ under $5 \% \mathrm{CO}_{2}$ until the solution turned significantly yellow. The optical density (OD) was measured at $450 \mathrm{~nm}$ on a microplate. The cell viability was calculated using the following formula: (OD of dosing well - OD of culture medium)/(OD of control well - OD of culture medium) $\times 100 \%$. The $50 \%$ inhibitory concentration (IC50) calculator (https://www.aatbio.com) was used to prepare the survival rate and drug concentration curves, and the best fitting IC50 was obtained. The statistical chart of the IC50 value was prepared using GraphPad prism 6 software. The following formula was used: Resistance index $(\mathrm{RI})=\mathrm{IC} 50$ of resistant cells/IC50 of parental cells.

\section{Western blot analysis and antibodies}

Western blot (WB) was performed as reported previously, ${ }^{17}$ and all the antibodies used are listed in supplementary table 1.

\section{Cellapoptosis analysis}

Flow cytometry was performed as reported previously, ${ }^{17}$ and the Annexin V Apoptosis Detection Kit APC was obtained from eBioscience.

\section{ROSdetection in cells and tissues}


CellROX Regent orange solution was used to culture or cover the tissue and avoid light during the whole process. After incubation for $30 \mathrm{~min}$ at $37^{\circ} \mathrm{C}$, we used a High Content Imaging System to observe ROS production in the cells. The dye solution was removed from the tissues and they were fixed. Then, photos were taken under a microscope, and the tissues were stored.

\section{Immunohistochemistry}

The tumor tissues were dehydrated and fixed in a $10 \%$ formalin buffer solution for $24 \mathrm{~h}$. The frozen sections were embedded in OCT, frozen, and continuously sectioned to $15 \mu \mathrm{m}$ sections at $20^{\circ} \mathrm{C}$. Immunohistochemistry was performed as previously described. The primary antibodies used are listed in Table S1.

\section{Animal models}

Parental A2058 cells or drug-resistant melanoma A2058R cells $\left(1 \times 10^{6}\right)$ in $200 \mu$ l of PBS were subcutaneously transplanted into SPF BALB/c nude mice (purchased from Zhongshan Hospital Laboratory). After 7-10 days of transplantation, the palpable tumor size (40-100 mm) was reached, and mice with a similar tumor size were randomly assigned to different four treatment groups. The parallel group design was adopted, and the mice were treated with $30 \mathrm{mg} / \mathrm{kg}$ vemurafenib ,and/or 3-MPA(SKF34288 hydrochloride, Cat\#320386-54-7) (MedChemExpress, USA), and equal DMSO,once a day for 21 days. All animal protocols were approved by the animal protection and the use committee of Zhongshan Hospital, Fudan University.

\section{Calculation of the combination index}

CompuSyn is available for download from http://www.combosyn.com. According to the Chou-Talalay mathematical model of drug interaction, the combination index $(\mathrm{Cl})$ of cells receiving combination therapy was calculated. Chou-Talalay's $\mathrm{Ci}$ theorem provides a quantitative definition of the additive effect $(\mathrm{Cl}=1)$, synergistic effect $(\mathrm{Cl}<1)$, and antagonistic effect $(\mathrm{Cl}>1)$ of drug combination ${ }^{17}$.

\section{Statistical analysis}

Each experiment was repeated three times. The results are shown as the mean \pm standard deviation. SPSS software 19.0 was used for the statistical analyses, including Student's t-test (two-tailed), Pearson's correlation analysis, and the Log-Rank test. The significance threshold was set at 0.05 for each test.

\section{Results}

\section{Establishment and phenotype of the drug resistance model.}


To investigate the molecular mechanisms underlying BRAFi resistance, we exposed the melanoma cell line A2058 harboring the BRAF V600E mutation to high concentrations of vemurafenib. After 6 months, we obtained the resistant cell line A2058R. We found that the cells became slender under the action of vemurafenib at a high concentration (Figure 1A, 1B). Results of the CCK8 experiment confirmed that the $\mathrm{IC}_{50}$ of drug-resistant A2058R cells was higher than that of primary A2058 cells, (A2058 IC50 $=0.71$, $\left.\mathrm{A} 2058 \mathrm{R} \mathrm{IC}_{50}=21.95, \mathrm{RI}=30.9, P<0.005\right)$ (Figure 1C, 1D). Scratch and Transwell assays confirmed that A2058R cells have greater invasion and migration abilities than A2058 cells (Figure 1E - 1I). Meanwhile, WB assays showed that the levels of CD271, SOX10, TWIST, and SLUG were elevated in A2058R cells, indicating that they possess higher stemness and EMT abilities.

\section{PCK1 protein and AKT/PI3K signaling were key factors in the transcriptome sequencing results.}

After normalizing the transcriptome sequencing results (Figure 2A), we found that there were differences in the gene expression profiles among the four samples (Figure 2B), which was further corroborated by PCA results (Figure 2C). Differential expression analysis was performed, and we focused on PCK1, a gene whose expression level is high and constant in R1, R2, and R3, and we considered it as the key target gene for conferring of drug resistance (Figure 2D). In KEGG enrichment analysis (Figure 2E), we observed that the PI3K/AKT signaling pathway molecules were continuously highly expressed in the three drugresistant samples. Our results in combination with the literature on this topic indicate that the PCK1 and $\mathrm{PI} 3 \mathrm{~K} /$ Akt signaling pathways are involved in the process of acquired drug resistance induced by BRAFi in melanoma cells. Furthermore, western blot analysis showed that the expression of the PCK1 and $\mathrm{PI} 3 \mathrm{~K} / \mathrm{Akt}$ signaling pathways was up-regulated and activated in drug-resistant cells. In addition, when we inhibited the PI3K/Akt pathway, the expression of PCK1 decreased, suggesting that PCK1 is a crucial downstream molecule for the PI3K/Akt signaling pathway for achievement of drug resistance (Figure 2F).

\section{PCK1 activity determined melanoma cell resistance to vemurafenib.}

Next, we constructed low expression and overexpression PCK1 cell lines using siRNA and overexpression viruses, respectively. Western blot results indicated that the transfection was successful (Figure 3A). After PCK1 knockdown, cell viability significantly reduced; thus, the IC50 of siPCK1 and the resistance index (RI) both decreased (RI = 4.46), $P<0.005$ (Figure 3B, 3C).

However, the cell viability and resistance index $(\mathrm{RI})$ were higher $(\mathrm{RI}=9.27), P<0.005$, in the cell line overexpressing PCK1 (Figure 3B, 3D). Therefore, the results indicated that PCK1 is a crucial molecule that can enhance the tolerance of melanoma cells to vemurafenib. 


\section{PCK1 promoted the proliferation, migration, and stemness of melanoma cells with V600E mutation.}

Based on the hypothesis that the development of drug resistance may be caused by related genes that induce cancer stemness, EMT, and apoptosis inhibition, we first designed scratch and invasion experiments and found that the PCK1 gene status was related to the proliferation and invasion of melanoma cells, while the activation of PCK1 could promote drug resistance in melanoma and increase the severity of the disease (Figure 4A, 4B). Next, flow cytometry was conducted, and the results showed that the knockdown and overexpression of PCK1 did not have a direct effect on apoptosis, although statistical differences were noted. This suggested that PCK1 might indirectly induce BRAFi resistance through PPP (Figure 4C-4G). In addition, the results of the tumor spheroidization test on a low adhesion plate showed that the spheroidization ability of A2058R cells was significantly higher than that of A2058 cells (Figure 4H), that of the SiPCK1 group was decreased, and that of the OE group was restored after the overexpression of PCK1. Combined with the western blot results, these results suggested that CD271 and SOX10, which have been recognized as indicators of melanoma stemness, were highly expressed in A2058R and OE cells (Figure 4I), indicating that PCK1 could modify cancer stemness in the development of drug resistance. Meanwhile, in this experiment, PCK1 could not alter the EMT of melanoma for achievement of drug resistance (Figure $4 \mathrm{~J}$ ). These lines of evidence illustrated that the activation of PCK1 potentiated melanoma proliferation, migration, and tumor stemness, all the phenotypes associated with drug resistance. The results revealed that PCK1 did not contribute to drug resistance directly by inhibiting apoptosis but, most likely, contributed indirectly to acquired drug resistance by triggering some of the cascades of the biochemical metabolic chain.

\section{Synergistic effect of PCK1 inhibitor 3-MPA combined with vemurafenib on drug-resistant cells.}

A CCK8 assay was used to detect the activity of A2058R cells under different drug treatment regimens to evaluate the advantages of a combined treatment strategy. The IC50 of A2058R cells treated with 3-MPA or vemurafenib alone did not change significantly (Figure 5A, 5C). However, the IC50 of 3-MPA combined with vemurafenib was significantly lower than that of vemurafenib alone, which indicated that 3-MPA increased the resistance inhibition of vemurafenib (Figure 5B, 5C). In order to evaluate the efficacy of the combination therapy, we used the Chou-Talalay method to compare the best fitting IC50 of each treatment group again. In the experiments in which drug-resistant A2058R cells were treated, the IC50 of the 3-MPA + vemurafenib group was 6.37 , that of vemurafenib monotherapy was 14.47 , that of 3-MPA monotherapy was 27.47 , and the combination index $(\mathrm{Cl})=0.3362<1$, which showed that the combination of 3-MPA and vemurafenib had a synergistic effect on pharmacology (Figure 5D, 5E). Moreover, only when the reaction part of the system reaches an $0.8<\mathrm{FA}<0.9$, the synergistic effect will be reversed to the antagonistic effect, which indicates that change in the doses of the two drugs has little effect on the synergistic effect and that this effect is stable (Figure 5F). 


\section{Inhibition of PCK1 by 3-MPA led to ROS accumulation and oxidative damage in drug-resistant cells.}

Since PCK1 is a key enzyme in the PPP process and PPP controls the intracellular ROS levels, the supplement of reduction equivalent is undoubtedly of great value to melanoma cells. Therefore, we speculated that the downstream mechanism of PCK1 might be related to the regulation of ROS levels. We used the High Content Live Cell Imaging System to observe the ROS levels. As shown in Figure 6A, we found that the ROS level in primary A2058 cells was significantly higher than that in A2058R cells (Figure $6 \mathrm{~B})$, which demonstrated that drug-resistant cells have a better ROS reduction ability. When A2058R cells were exposed to vemurafenib, the ROS level was still lower than that in A2058 cells. In addition, the ROS levels of primary A2058 cells and drug-resistant A2058R cells were higher than those in A2058R cells after adding 3-MPA. When A2058 and A2058R cells were treated with a combination of 3-MPA and vemurafenib, the ROS level was increased significantly, which suggested that the ROS level of drugresistant cells was determined by the activity of PCK1.

We generated a subcutaneous xenograft model from nude mice, forming cryosections that were analyzed using a ROS probe (Figure 6C). Combined with the tumor volume/wt (Figure 7D, 7E), we found that the ROS levels were higher in the A2058 + vemurafenib group than in the A2058 + DMSO group, whereas the number of tumor cells was reduced relative to the A2058 + DMSO group, indicating that vemurafenib inhibited V600E mutated melanoma progression by generating strong oxidative damage. Compared with the A2058R + vemurafenib group, the A2058 + vemurafenib group exhibited higher intracellular ROS levels and decreased tumor cell numbers, indicating that the drug-resistant cells hedge the killing effect of vemurafenib via metabolic reprogramming. Compared with the A2058R + vemurafenib group, the A2058R + 3-MPA + vemurafenib group showed a significant increase in ROS levels and a decrease in the number of tumor cells, demonstrating that, in drug-resistant cells, the inhibition of PCK1 sensitizes melanoma to BRAFi inhibition. The increase in ROS levels was mainly caused by the obstruction of the PPP pathway. However, when we added 3-MPA $(20 \mu \mathrm{mol} / \mathrm{l})$ to A2058 and A2058R cells, the WB results showed that the expression of KEAP1 was increased after PCK1 silencing, which indicated that PCK1 inhibited the expression of KEAP1 (Figure 6D). These pieces of evidence all pointed the spearhead of resistance toward ROS reduction, which was the result of PCK1 activity. 3-MPA blocked PCK1, thereby enabling ROS accumulation and suppressing chemoresistance in melanoma cells.

\section{3-MPA improved the inhibitory effect of BRAFi in vivo}

Next, we evaluated the effect of combination therapy in melanoma using a subcutaneous xenograft mouse model (Figure 7A, 7B). According to the wt and volume data (Figure 7C, 7D), we found that the combination of vemurafenib and 3-MPA in the drug-resistant group could reverse the drug-resistant situation and significantly reduce the wet and volume of the tumor tissue. Compared with A2058 primary melanoma cells, the inhibition ability of the combination group was even more prominent, which suggested that the combination of BRAFi and PCK1 could achieve an excellent inhibition effect in BRAFi- 
resistant cells with PCK1 mutation. Combined with the immunohistochemical results, as mentioned above, we further confirmed that the main effect of 3-MPA was to increase the ROS levels of melanoma cells, disrupt the oxidative balance in drug-resistant melanoma cells, and expose them to an oxidative attack again.

The immunohistochemistry results showed that in the subcutaneous tumor tissues of nude mice inoculated with primary A2058 cells, the expression of PCK1 in the vemurafenib group was up-regulated compared with the DMSO group, while KEAP1 was inhibited (Figure 6D). In the tissues of A2058R cells, the up-regulated expression of PCK1 in the group treated with vemurafenib in combination with 3-MPA was limited compared with that in the vemurafenib group, but the expression of KEAP1 was up-regulated. Therefore, both the in vitro and in vivo experiments confirmed that vemurafenib could induce high expression of PCK1, and then inhibit the synthesis of KEAP1, thus inducing drug resistance. However, when 3-MPA was used to inhibit PCK1, KEAP1 was also activated and ROS accumulated in the cells, which made the drug-resistant melanoma cells suffer from oxidative attack and inhibited tumor progression.

\section{Discussion}

Acquired resistance is the main clinical obstacle to improving the prognosis of melanoma patients, and the known mechanisms involve various components. ${ }^{18,19}$ The most common pathological mechanisms are the reactivation of the BRAF/MEK pathway or other proliferation promoting signal transduction pathways, the increase in the BRAF V600E protein copy number, RAS mutations, and the activation of ARAF and CRAF, which promote the formation of a dimer that BRAFi cannot inhibit. ${ }^{20,21,22}$ Moreover, the activation and mutation of MEK1/MEK2 and the high expression of COT lead to the activation of the MAPK/ERK pathway downstream of BRAF. ${ }^{23}$ Overexpressed RTKs and the compensatory activation of the Akt/PI3K/mTOR pathway contribute to drug resistance. ${ }^{24,25}$ Our study also demonstrated that the adaptive hyperactivation of AKT in response to BRAFi compensated for BRAF inactivation, and the mutually compensatory relationship between the Akt/PI3K/mTOR signal transduction pathway and the ERK pathway supported tumor adaptation and resistance to specific interventions. Therefore, we concluded that the AKT/PI3K pathway was an extremely important signaling axis, promoting cell proliferation, migration, and stemness, which contributed to BRAFi resistance in V600E mutated melanoma.

Although there are many downstream effectors of PI3K and Akt that can change the function of melanoma cells, PCK1 synthesis and activation are important processes mediated after AKT phosphorylation. The Akt/PI3K/mTOR pathway was mainly related to the mechanism of metabolic reprogramming, ${ }^{26}$ including the activation of mTOR complex 1 (mTORC1), glycogen synthase kinase 3 (GSK3), and forkhead box 0 (FoxO) transcription factor family members. ${ }^{27,28}$ In our study, the activity of the Akt/PI3K/mTOR pathway and the TPCK1 levels always paralleled each other in vitro, probably because the activation of mTORC1 molecules induced cytosolic SREBP to promote PCK1 synthesis. ${ }^{29}$ 
Additionally, Fox01 deacetylation synergizes PGC-1a action to promote PCK1 transcription. It has recently been reported that activated Akt can directly phosphorylate PCK1 at Ser-90. ${ }^{27,30}$ Ultimately, the biological processes mediated by PCK1 under the agonism of AKT are critical steps for melanoma cells to survive and proliferate.

A redox homeostasis imbalance elevates ROS levels and is pivotal in driving the processes of carcinogenesis, metastasis, and drug resistance in cutaneous melanoma. ${ }^{31,32,33}$ Lim pointed out that was the process of redox metabolism rewiring in melanoma cells. ${ }^{34}$ During the course of long-term treatment with BRAFi, the upregulated MITF-PGC1a axis and the long non-coding RNA (IncRNA) SAMMSON-p32 led to an inevitable accumulation of ROS. ${ }^{35}$ High levels of ROS should have induced apoptosis, but genetic changes led cells to survive at high levels of ROS by increasing the NADPH level through the pentose phosphate pathway (PPP) or by activating the

KEAP1/Nrf2 antioxidant signaling pathway. ${ }^{36,37}$ One of the crucial switches driving redox remodeling was PCK1, which is a key enzyme involved in the PPP process, ${ }^{38}$ playing a role in the antioxidant function of tumors. ${ }^{39}$ This requires the presence of PCK1 to synthesize abundant reductive NADPH via the PPP, and its inhibition impedes melanoma growth. ${ }^{40,41}$ Moreover, PCK1 repressed KEAP1 and the Nrf2 restriction was removed, further activating the antioxidant repair ability of cells. Thus, a large amount of reductive NADPH was produced, which counteracted the cell damage induced by high levels of ROS and enabled cells no close to achieving redox equilibrium to survive.

Studies on the role of redox fragility in melanoma progression have been reported, and BRAFi-resistant melanoma cells with elevated ROS levels are more sensitive to the death induced by oxidants. ${ }^{42,48}$ This is highly consistent with our results, which showed that 3-MPA sensitized the biological effect of vemurafenib to restore the sensitivity of tumors. When 3-MPA was used, drug-resistant cells lost the ability of supplementing the reduction equivalent, the redox level was unbalanced again, and the cells experienced oxidative damage. The specific mechanism underlying this effect might be that 3-MPA blocks the PPP pathway to synthesize NADPH and nucleic acids, inhibiting cell proliferation and leading to the accumulation of intracellular ROS. In addition, interestingly, the increase in ROS level should play a protective role through the KEAP1/Nrf2 axis. However, when 3-MPA was used, the expression of KEAP1, a repressor of Nrf2, increased. ${ }^{49}$ As the KEAP1/Nrf2 axis had cellular protective effects on the antioxidant and detoxification activities, ${ }^{50}$ inhibiting Nrf2 undoubtedly aggravated the oxidative burden of tumor cells. These results suggested that 3-MPA not only down-regulates the PPP pathway, but also indirectly up-regulates KEAP1, leading to a loss of the self-antioxidant repair ability of drug-resistant cells; as a result, the combination of 3-MPA and vemurafenib resulted in outstanding therapeutic effects.

One limitation of our study is that only the melanoma A2058 cell line was used for modeling. Moreover, the drug resistance was a relatively long-term change, and the observation time used in our in vitro and in vivo experiments was relatively short. Therefore, the role of PCK1 in the drug resistance process of other 
tumor cells needed to be further revealed, and the observation cycle also needs to be optimized to explore the specific functions of PCK1 from a long-term and objective perspective.

\section{Conclusions}

Oue study found that the activation of the Akt/PI3K/mTOR signaling pathway compensated for the tumor promoting effect in the BRAFi-resistant melanoma cells. Akt/PI3K upregulated PCK1, which turned on the PPP switch and inhibited KEAP1, and then synthesized enough reductive equivalent to decrease the ROS levels(Figure 8),leading to BRAFi resistance. PCK1 also promoted cell proliferation, migration, and tumor stemness. As AKT/PI3K/mTOR pathway widely distributed, patients with direct suppression of it may have more side effects. Based on the strategy of precision treatment,we used an antihyperglycemic agent 3-MPA combined with vemurafenib and found it enhanced the sensitivity of drugs obviously.Finally, we clarified the mechanism of resistance to BRAFi and provided new options for clinically targeted combination therapy in patients with advanced melanoma.

\section{Abbreviations}

Kelch-like ECH-associated protein 1 KEAP1

Pentose Phosphate Pathway PPP

3-Mercaptopicolinic Acid 3-MPA

Phosphoenolpyruvate carboxykinase 1 PCK1

Reactive Oxygen Species ROS

Epithelial-to-Mesenchymal Transition EMT

CellCounting Kit 8 CCK8

Tricarboxylic Acid Cycle TCA

Optical Density OD

Half Inhibitory Concentration 50 IC50

phosphate buffered solution PBS

Combination Index $\mathrm{Cl}$

\section{Declarations}




\section{Consent for publication}

Not applicable

\section{Availability of data and materials}

The datasets used and/or analysed during the current study are available from the corresponding author on reasonable request.

\section{Competing interests}

The authors declare that they have no competing interests

\section{Funding}

The project was supported by the National Natural Science Foundation of China.

\section{Authors' contributions}

MR,CYW and JYG came up with the hypothesis and designed the experiments. MR,XYD and MYT performed cell experiments. ZXG and QW performed the animal experiments. KJS,YTD and LQQ interpreted the data. MR and LW wrote the manuscript. JYG and CYW supervised the overall research, secured funding, and interpreted results.All authors read and approved the final manuscript.

\section{Acknowledgements}

Not applicable

\section{References}

1. Wei CY, Zhu MX, Yang YW, Zhang PF, Yang X, Peng R, Gao C, Lu JC, Wang L, Deng XY, Lu NH, Qi FZ, Gu JY. Downregulation of RNF128 activates Wnt/ $\beta$-catenin signaling to induce cellular EMT and stemness via CD44 and CTTN ubiquitination in melanoma. J Hematol Oncol. 2019 Mar 4;12(1):21. doi: 10.1186/s13045-019-0711-z. PMID: 30832692; PMCID: PMC6399928.

2. Davies H, Bignell GR, Cox C, et al. Mutations of the BRAF gene in human cancer. Nature. 2002;417(6892):949-954. 
3. Long, G. V. et al. Prognostic and clinicopathologic associations of oncogenic BRAF in metastatic melanoma. J. Clin. Oncol. 29, 1239-1246 (2011).

4. Jemal A,Seigel R,Ward E,et al.Cancer Statistics,2006[J].CA Cancer J Clin,2006,56(8):106-130.

5. Flaherty, K. T. et al. Combined BRAF and MEK inhibition in melanoma with BRAF V600 mutations. N. Engl. J. Med. 367, 1694-1703 (2012).

6. Robert, C. et al. Two year estimate of overall survival in COMBI-v, a randomized, open-label, phase III study comparing the combination of dabrafenib (D) and trametinib (T) with vemurafenib (Vem) as first-line therapy in patients (pts) with unresectable or metastatic BRAF V600E/K mutation-positive cutaneous melanoma [abstract 3301]. Eur. J. Cancer51 (Suppl. 3), S663 (2015).

7. Flaherty, K. T. et al. Combined BRAF and MEK inhibition in melanoma with BRAF V600 mutations. N. Engl. J. Med. 367, 1694-1703 (2012).

8. Villanueva J, Vultur A, Lee JT, Somasundaram R, Fukunaga-Kalabis M, Cipolla AK, et al. Acquired resistance to BRAF inhibitors mediated by a RAF kinase switch in melanoma can be overcome by cotargeting MEK and IGF-1R/PI3K. Cancer Cell 2010; 18(6):683-695. [PubMed: 21156289]

9. Schadendorf D, Long GV, Stroiakovski D, Karaszewska B, Hauschild A, Levchenko E, Chiarion-Sileni V, Schachter J, Garbe C, Dutriaux C, Gogas H, Mandalà M, Haanen JBAG, Lebbé C, Mackiewicz A, Rutkowski P, Grob JJ, Nathan P, Ribas A, Davies MA, Zhang Y, Kaper M, Mookerjee B, Legos JJ, Flaherty KT, Robert C. Three-year pooled analysis of factors associated with clinical outcomes across dabrafenib and trametinib combination therapy phase 3 randomised trials. Eur J Cancer. 2017 Sep;82:45-55. doi: 10.1016/j.ejca.2017.05.033. Epub 2017 Jun 22. PMID: 28648698.

10. Avagliano A., Fiume G., Pelagalli A., Sanita G., Ruocco M.R., Montagnani S., Arcucci A. Metabolic plasticity of melanoma cells and their crosstalk with tumor microenvironment. Front. Oncol. 2020;10:1-21. doi: 10.3389/fonc.2020.00722.

11. Montal, E.D. et al. (2015) PEPCK coordinates the regulation of central carbon metabolism to promote cancer cell growth. Mol. Cell 60, 571-583.

12. Li, Y. et al. (2015) Upregulation of cytosolic phosphoenolpyr- uvate carboxykinase is a critical metabolic event in melanoma cells that repopulate tumors. Cancer Res. 75, 1191-1196

13. Wang Z, Dong C. Gluconeogenesis in Cancer: Function and Regulation of PEPCK, FBPase, and G6Pase. Trends Cancer. 2019 Jan;5(1):30-45. doi: 10.1016/j.trecan.2018.11.003. Epub 2018 Dec 20. PMID: 30616754.

14. Nordlie RC, Lardy HA. Mammalian liver Phosphoenolpyruvate Carboxykinase activities. J Biol Chem. 1963;238:2259-2263. [PubMed] [Google Scholar]

15. Wang Z, Dong C. Gluconeogenesis in Cancer: Function and Regulation of PEPCK, FBPase, and G6Pase. Trends Cancer. 2019 Jan;5(1):30-45. doi: 10.1016/j.trecan.2018.11.003. Epub 2018 Dec 20. PMID: 30616754.

16. Ma R, Ji T, Zhang H, Dong W, Chen X, Xu P, Chen D, Liang X, Yin X, Liu Y, Ma J, Tang K, Zhang Y, Peng Y, Lu J, Zhang Y, Qin X, Cao X, Wan Y, Huang B. A Pck1-directed glycogen metabolic program 
regulates formation and maintenance of memory CD8+ T cells. Nat Cell Biol. 2018 Jan;20(1):21-27. doi: 10.1038/s41556-017-0002-2. Epub 2017 Dec 11. PMID: 29230018.

17. Wang D, Yu D, Liu X, Wang Q, Chen X, Hu X, Wang Q, Jin C, Wen L, Zhang L. Targeting laryngeal cancer cells with 5-fluorouracil and curcumin using mesoporous silica nanoparticles. Technol Cancer Res Treat. 2020 Jan-Dec;19:1533033820962114. doi: 10.1177/1533033820962114. PMID: $33267716 ;$ PMCID: PMC7720313.

18. Sun C, Wang L, Huang S, Heynen GJ, Prahallad A, Robert C, Haanen J, et al. Reversible and adaptive resistance to BRAF(V600E) inhibition in melanoma. Nature 2014; 508(7494):118-122. [PubMed: 24670642]

19. Gide TN, Wilmott JS, Scolyer RA, Long GV. Primary and Acquired Resistance to Immune Checkpoint Inhibitors in Metastatic Melanoma. Clin Cancer Res. 2018 Mar 15;24(6):1260-1270. doi: 10.1158/1078-0432.CCR-17-2267. Epub 2017 Nov 10. PMID: 29127120.

20. Chen G, Davies MA. Targeted therapy resistance mechanisms and therapeutic implications in melanoma. Hematol Oncol Clin North Am 2014; 28(3):523-536. [PubMed: 24880945]

21. Bartnik E., Fiedorowicz M., Czarnecka A.M. Mechanisms of Melanoma Resistance to Treatment with BRAF and MEK Inhibitors. Nowotwory J. Oncol. 2019;69:133-141. doi: 10.5603/NJ0.2019.0025.

22. Patel H., Yacoub N., Mishra R., White A., Long Y., Alanazi S., Garrett J.T. Current Advances in the Treatment of BRAF-Mutant Melanoma. Cancers. 2020;12:482. doi: 10.3390/cancers12020482.

23. Sharma V., Young L., Cavadas M., Owen K., Reproducibility Project: Cancer Biology Registered Report: COT drives resistance to RAF inhibition through MAP kinase pathway reactivation. Elife. 2016;5:e11414. doi: 10.7554/eLife.11414.

24. Chan X.Y., Singh A., Osman N., Piva T.J. Role Played by Signalling Pathways in Overcoming BRAF Inhibitor Resistance in Melanoma. Int. J. Mol. Sci. 2017;18:1527. doi: 10.3390/ijms18071527.

25. Caporali S., Alvino E., Lacal P.M., Levati L., Giurato G., Memoli D., Caprini E., Antonini Cappellini G.C., D'Atri S. Targeting the PI3K/AKT/mTOR pathway overcomes the stimulating effect of dabrafenib on the invasive behavior of melanoma cells with acquired resistance to the BRAF inhibitor. Int. J. Oncol. 2016;49:1164-1174. doi: 10.3892/ijo.2016.3594.

26. Manning BD, Toker A. AKT/PKB Signaling: Navigating the Network. Cell. 2017 Apr 20;169(3):381-405. doi: 10.1016/j.cell.2017.04.001. PMID: 28431241; PMCID: PMC5546324.

27. Manning BD, Toker A. AKT/PKB Signaling: Navigating the Network. Cell. 2017 Apr 20;169(3):381-405. doi: 10.1016/j.cell.2017.04.001. PMID: 28431241; PMCID: PMC5546324.

28. Webb AE, Brunet A. FOXO transcription factors: key regulators of cellular quality control. Trends Biochem Sci. 2014;39:159-169.

29. Yi J, Zhu J, Wu J, Thompson CB, Jiang X. Oncogenic activation of PI3K-AKT-mTOR signaling suppresses ferroptosis via SREBP-mediated lipogenesis. Proc Natl Acad Sci U S A. 2020 Dec 8;117(49):31189-31197. doi: 10.1073/pnas.2017152117. Epub 2020 Nov 23. PMID: 33229547; PMCID: PMC7733797. 
30. Xu D, Wang Z, Xia Y, Shao F, Xia W, Wei Y, Li X, Qian X, Lee JH, Du L, Zheng Y, Lv G, Leu JS, Wang H, Xing D, Liang T, Hung MC, Lu Z. The gluconeogenic enzyme PCK1 phosphorylates INSIG1/2 for lipogenesis. Nature. 2020 Apr;580(7804):530-535. doi: 10.1038/s41586-020-2183-2. Epub 2020 Apr 8. PMID: 32322062.

31. de Jager T.L., Cockrell A.E., Du Plessis S.S. Ultraviolet light induced generation of reactive oxygen species. Adv. Exp. Med. Biol. 2017;996:15-23. doi: 10.1007/978-3-319-56017-5_2

32. Tsoi J. Multi-stage differentiation defines melanoma subtypes with differential vulnerability to druginduced iron-dependent oxidative stress. Canc. Cell. 2018;33:890-904. doi: 10.1016/j.ccell.2018.03.017. e895.

33. Cannavò S.P. The role of oxidative stress in the biology of melanoma: a systematic review. Pathol. Res. Pract. 2019;215:21-28. doi: 10.1016/j.prp.2018.11.020.

34. Corazao-Rozas P. Mitochondrial oxidative stress is the Achille's heel of melanoma cells resistant to Braf-mutant inhibitor. Oncotarget. 2013;4:1986-1998. doi: 10.18632/oncotarget.1420.

35. Haq R, Fisher DE, Widlund HR. Molecular pathways: BRAF induces bioenergetic adaptation by attenuating oxidative phosphorylation. Clin Cancer Res. 2014 May 1;20(9):2257-63. doi: 10.1158/1078-0432.CCR-13-0898. Epub 2014 Mar 7. PMID: 24610826; PMCID: PMC4008642.

36. Hayes JD, Dinkova-Kostova AT, Tew KD. Oxidative Stress in Cancer. Cancer Cell. 2020 Aug 10;38(2):167-197. doi: 10.1016/j.ccell.2020.06.001. Epub 2020 Jul 9. PMID: 32649885; PMCID: PMC7439808.

37. Park J-W, Kim SC, Kim WK, Hong JP, Kim K-H, Yeo HY, et al. Expression of phosphoenolpyruvate carboxykinase linked to chemoradiation susceptibility of human colon cancer cells. BMC Cancer. 2014 [cited 2018 May 13];14.

38. Vieira P, Cameron J, Rahikkala E, Keski-Filppula R, Zhang L-H, Santra S, et al. Novel homozygous PCK1 mutation causing cytosolic phosphoenolpyruvate carboxykinase deficiency presenting as childhood hypoglycemia, an abnormal pattern of urine metabolites and liver dysfunction. Mol Genet Metab. 2017;120:337-341. doi: 10.1016/j.ymgme.2017.02.003.

39. Tuo L, Xiang J, Pan X, Hu J, Tang H, Liang L, Xia J, Hu Y, Zhang W, Huang A,Wang K, Tang N.PCK1 negatively regulates cell cycle progression and hepatoma cell proliferation via the AMPK/p27Kip1 axis. J Exp Clin Cancer Res. 2019 Feb 4;38(1):50.doi:10.1186/s13046-019-1029-y.

40. Li Y, Luo S, Ma R, Liu J, Xu P, Zhang H, et al. Upregulation of cytosolic phosphoenolpyruvate carboxykinase is a critical metabolic event in melanoma cells that repopulate tumors. Cancer Res. 2015;75:1191-1196. doi: 10.1158/0008-5472.CAN-14-2615.

41. Montal ED, Dewi R, Bhalla K, Ou L, Hwang BJ, Ropell AE, Gordon C, Liu WJ, DeBerardinis RJ, Sudderth $\mathrm{J}$, Twaddel $\mathrm{W}$, et al. PEPCK coordinates the regulation of central carbon metabolism to promote cancer cell growth. Mol Cell. 2015;60:571-583.

42. Tsoi J. Multi-stage differentiation defines melanoma subtypes with differential vulnerability to druginduced iron-dependent oxidative stress. Canc. Cell. 2018;33:890-904. doi: 10.1016/j.ccell.2018.03.017. e895. 
43. Obrador E, Liu-Smith F, Dellinger RW, Salvador R, Meyskens FL, Estrela JM. Oxidative stress and antioxidants in the pathophysiology of malignant melanoma. Biol Chem. 2019 Apr 24;400(5):589612. doi: 10.1515/hsz-2018-0327. PMID: 30352021.

44. Itoh K, Wakabayashi N, Katoh Y, Ishii T, Igarashi K, Engel JD, Yamamoto M. Keap1 represses nuclear activation of antioxidant responsive elements by Nrf2 through binding to the amino-terminal Neh2 domain. Genes Dev. 1999 Jan 1;13(1):76-86. doi: 10.1101/gad.13.1.76. PMID: 9887101; PMCID: PMC316370.

45. Cuadrado A, Rojo Al, Wells G, Hayes JD, Cousin SP, Rumsey WL, Attucks OC, Franklin S, Levonen AL, Kensler TW, Dinkova-Kostova AT. Therapeutic targeting of the NRF2 and KEAP1 partnership in chronic diseases. Nat Rev Drug Discov. 2019 Apr;18(4):295-317. doi: 10.1038/s41573-018-0008-x. PMID: 30610225.

\section{Figures}


$\mathbf{A}$

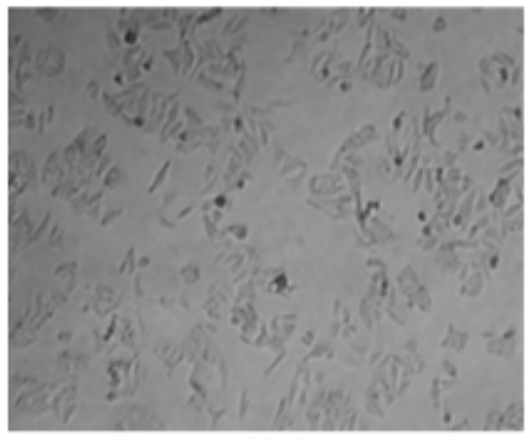

A2058

C

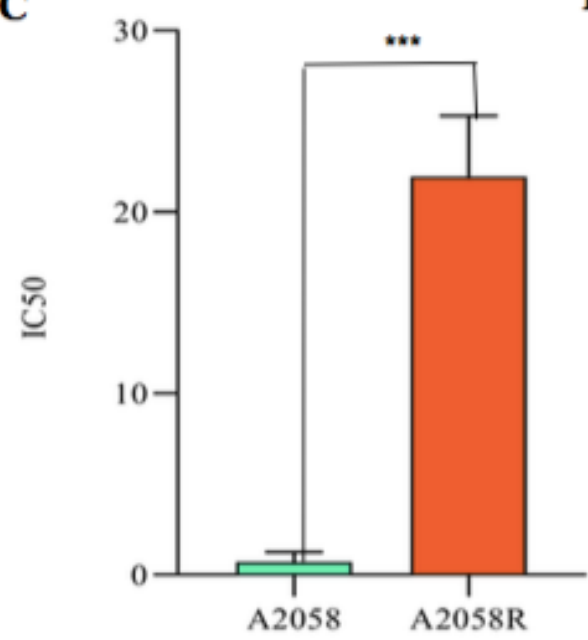

B

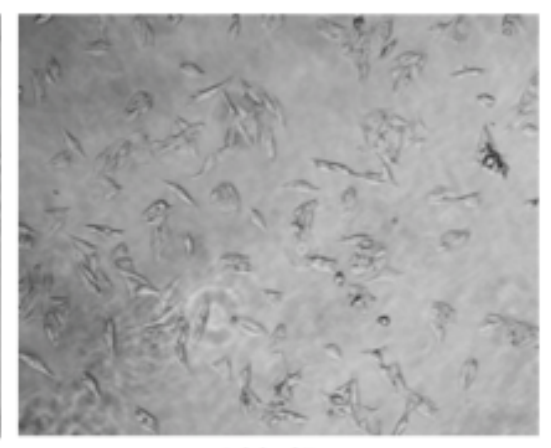

A2058R

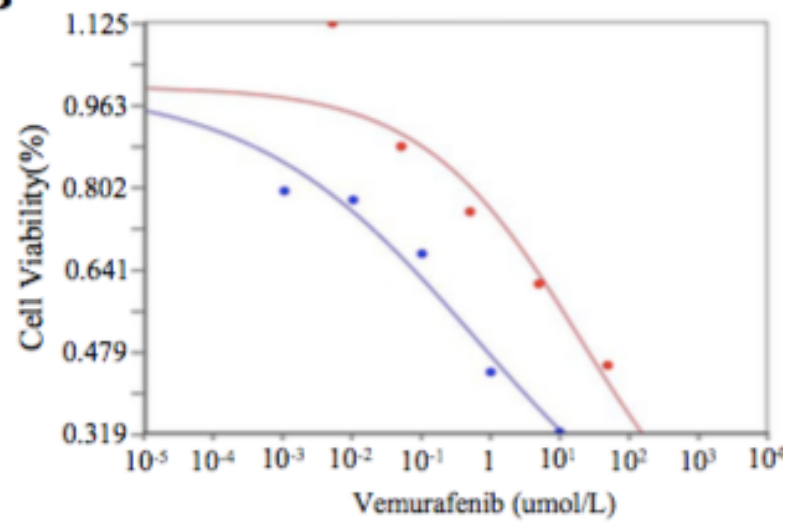

D

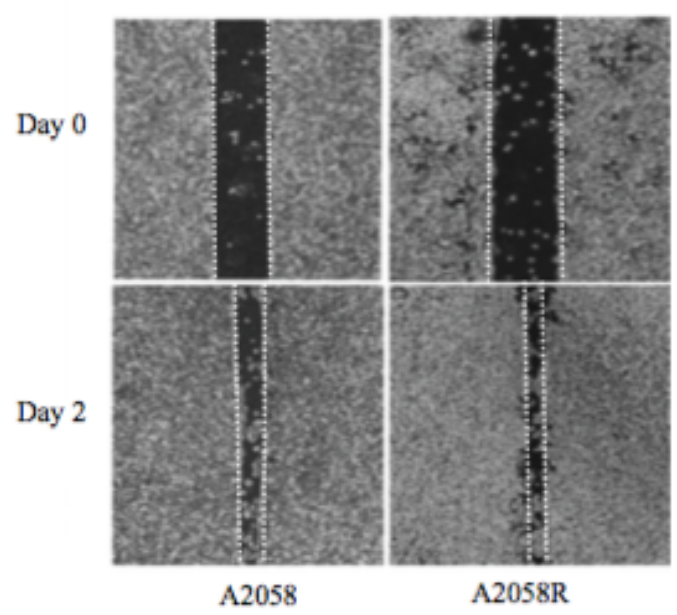

H

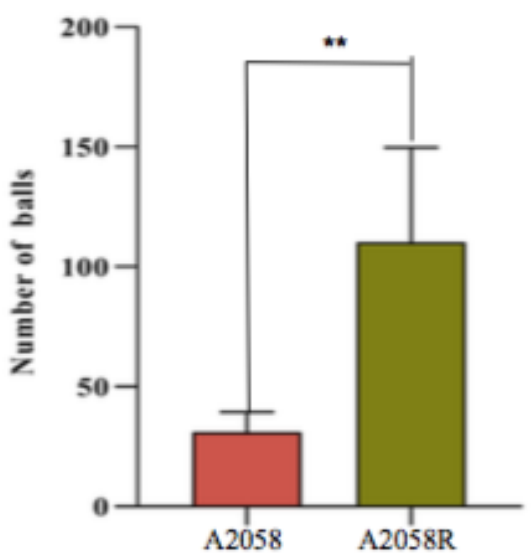

$\mathbf{E}$

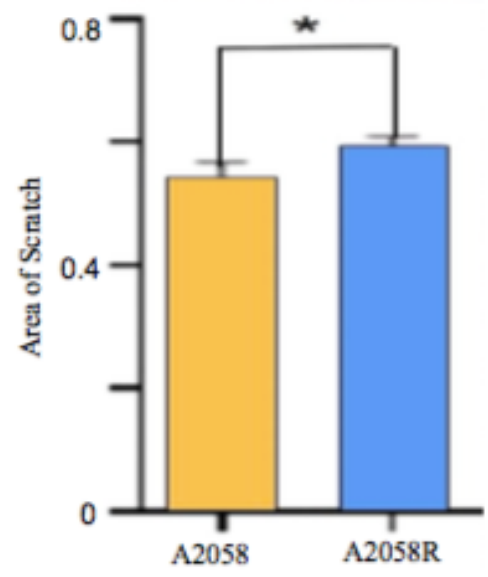

I

CD271

SOX10

GAPDH

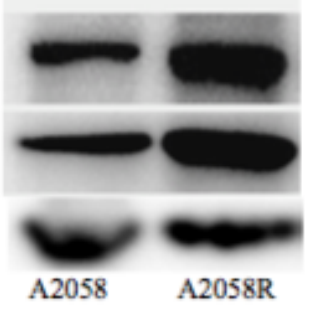

TWIST

SLUG

GAPDH

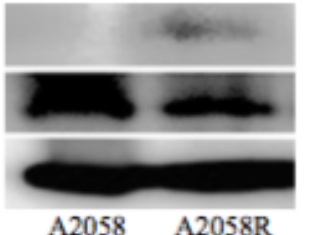

\section{Figure 1}

Establishment of a drug resistance model and the results of a phenotype test. A. Wild type A2058 cells and A2058R cells resistant to vemurafenib. B. Cell viability curve: the $x$-axis represents the vemurafenib concentration gradient, the $y$-axis represents the cell viability, the blue dots and lines represent A2058 cells, and the red represent A2058R cells. C. IC50 statistical plot: the y-axis represents the IC50, the difference between A2058 and A2058R cells was statistically significant, P < 0.05 . D. Scratch assay: day 0 refers to the width of the scratch $24 \mathrm{~h}$ after plating; day 2 refers to the width observed 2 days after 
scratching. E. Statistical figure of the scratch: the $y$-axis refers to the area of change before and after scratching, the difference between A2058 and A2058R cells was statistically significant, $P<0.05$. F. Transwell assay of A2058 cells. G. Transwell assay of A2058R cells. H. Results of the Transwell assay: the $y$-axis refers to the number of transmembrane cells. I. Sphere forming experiments: the $y$-axis refers to the number of cell spheres with a diameter greater than $50 \mu \mathrm{m}$ in each field, and the difference in the number of A2058 and A2058R spheres was statistically significant, $P<0.05$. J. Expression of stemness and EMT proteins: A2058R cells showed a higher expression of stemness proteins than A2058 cells, but there were no differences in the expression of EMT proteins.

$\mathbf{A}$

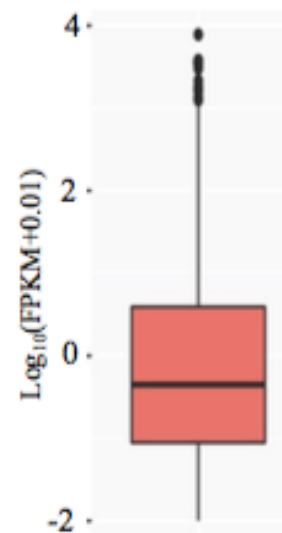

C

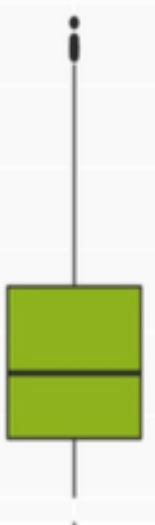

A2058_R0 A2058_R1 A2058_R2 A2058_R3

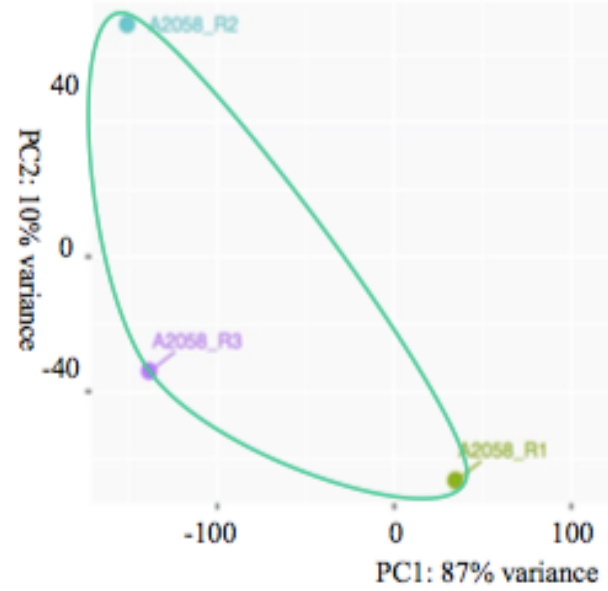

$\mathbf{E}$

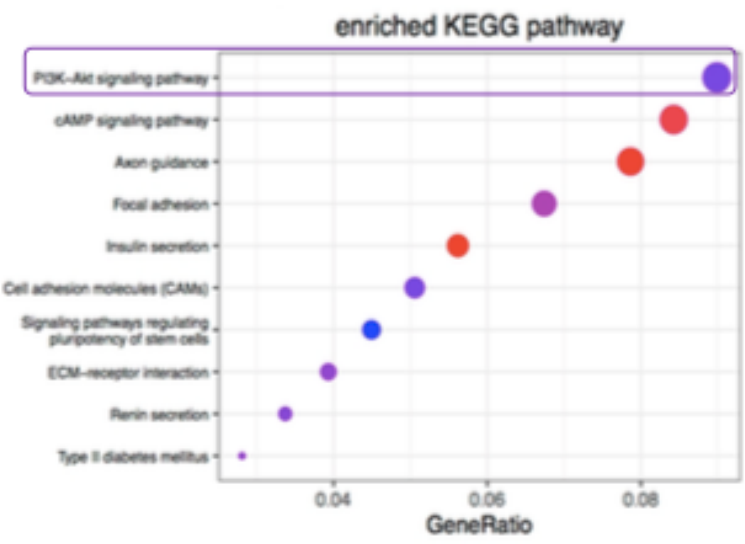

B

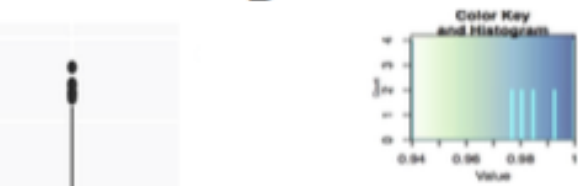

The pearson correlation of each
sample

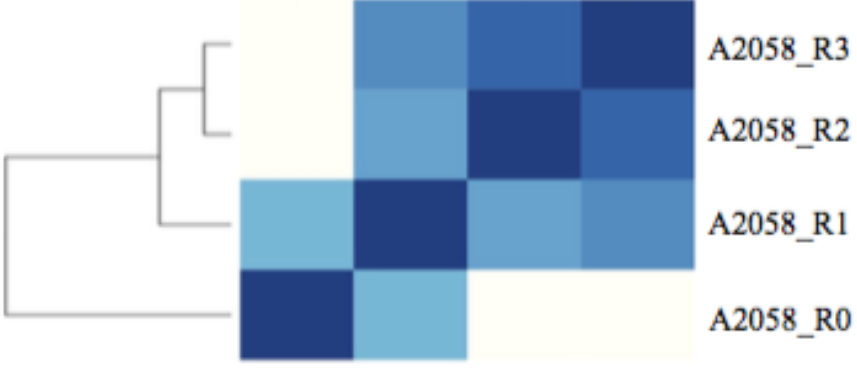

D
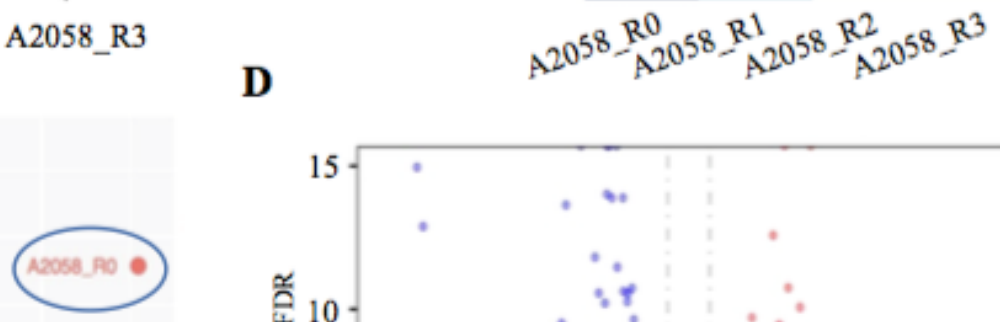

200

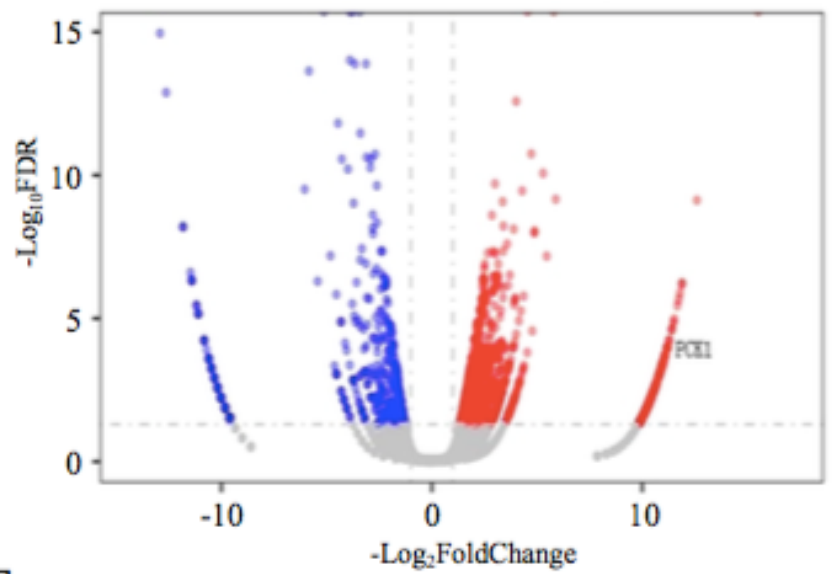

-Down

Not

Up
F

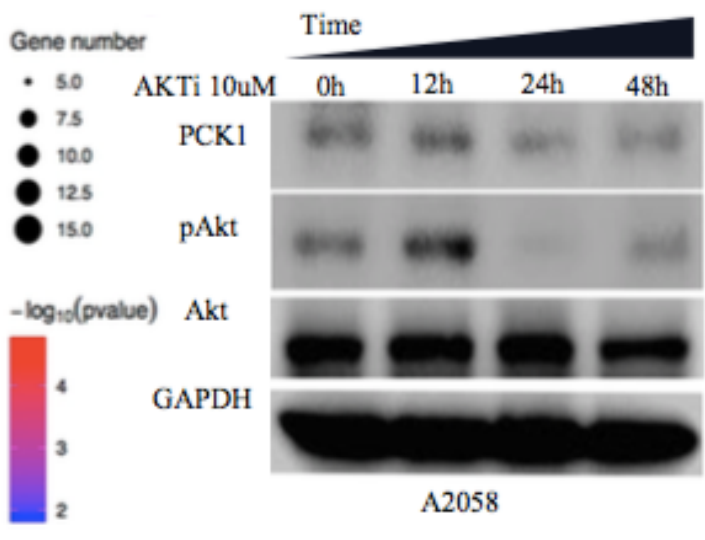

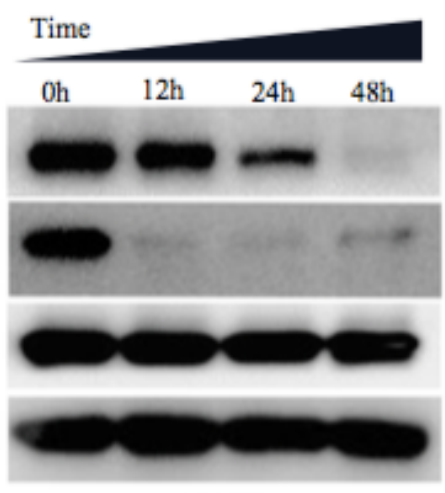

A2058R 


\section{Figure 2}

Identification and validation of the target molecules and signaling pathways using transcriptome sequencing. A. Standardized statistical plot: R0 represents wild-type sample; R1, R2, and R3 represent the samples collected during the construction of the resistant cell lines (refer to the Methods section). B. Pearson test: Pearson correlation in each chemoresistant sample suggested that the gene expression was variable for each sample. C. PCA analysis plot: the expression data were constitutively different between the wild-type and the selected cell lines receiving vemurafenib. D. Volcano plot: the genes presented in red are those that were up-regulated 2-fold and those presented in blue are the downregulated genes. The higher the position, the greater the difference. E. KEGG enrichment analysis plot: the size of the circle represents the number of related genes, and the larger the number, the higher is the number of genes. The color represents the statistical difference, with red being the most significant statistical difference. F. Validation of the signaling pathway: the expression level of PCK1 in A2058R cells is higher than that in A2058 cells, and the pAKT level in A2058R cells is higher than that in A2058 cells, indicating that AKT was activated in A2058R cells. After the addition of AKT inhibitor, the pAKT level gradually decreased, accompanied by the decrease in the PCK1 expression.

$\mathbf{A}$

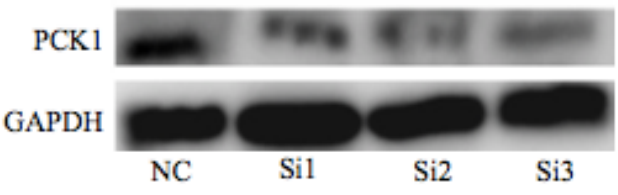

C

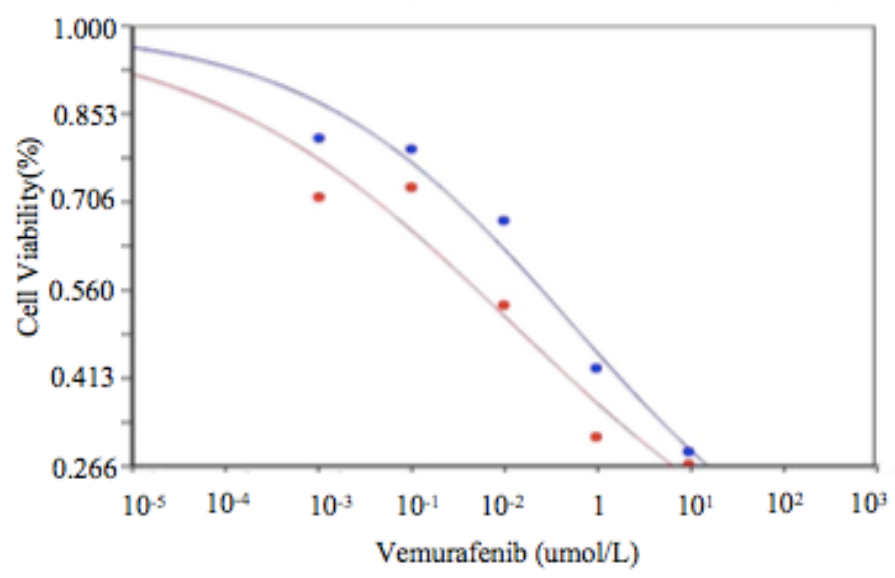

B

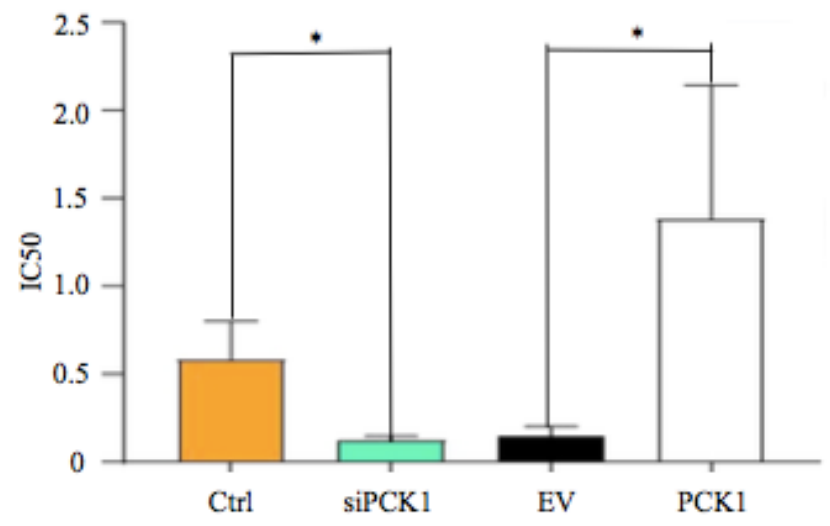

D

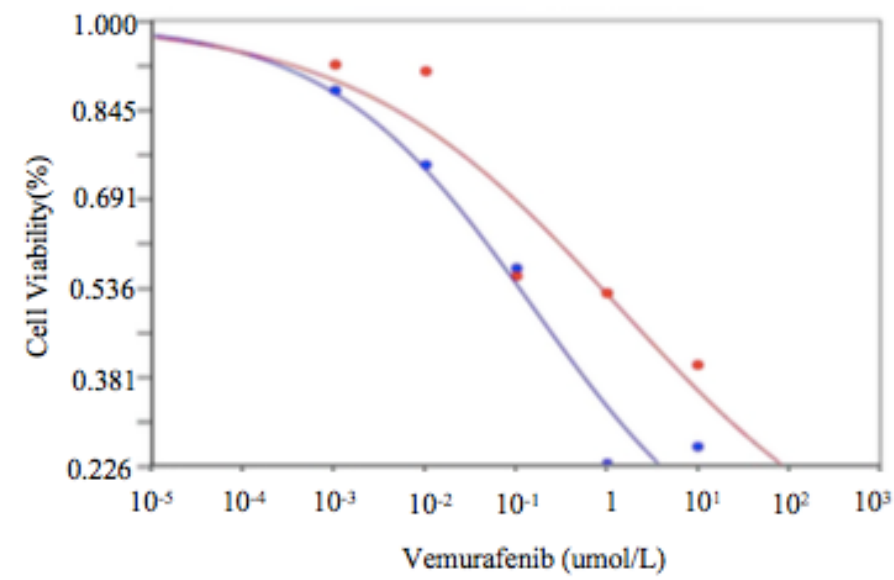

\section{Figure 3}


Effect of PCK1 on the chemoresistance in melanoma cells. A. Expression of PCK1: the melanoma cell lines with low expression and overexpression of PCK1 were constructed successfully. B. IC50 statistical figure: the IC50 of the cell line siPCK1 expressing low levels of PCK1 in vemurafenib decreased, and that of the high expression cell line in vemurafenib increased. C. Cell viability curve: the blue dots and lines refer to the control, and the red dots and lines refer to the cells that were used for the siRNA experiment (siPCK1). D. Cell viability curve: the blue dots and lines represent the empty virus (EV), and the red represents the overexpression of PCK1 (PCK1).

A

Day 0

Day 2

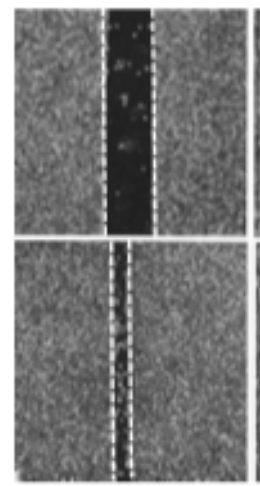

Ctrl

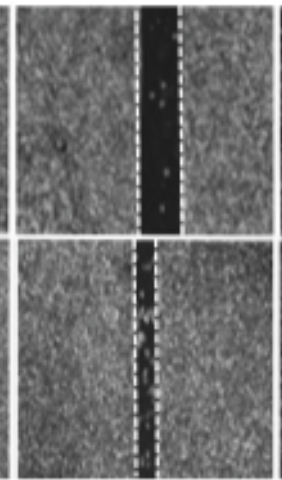

siPCK1

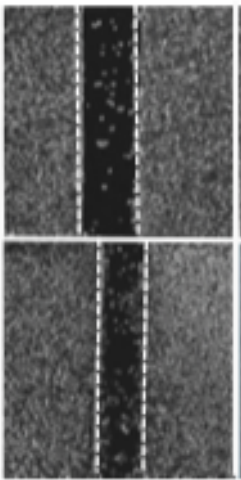

EV

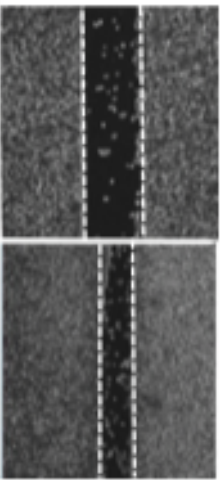

PCK1
C

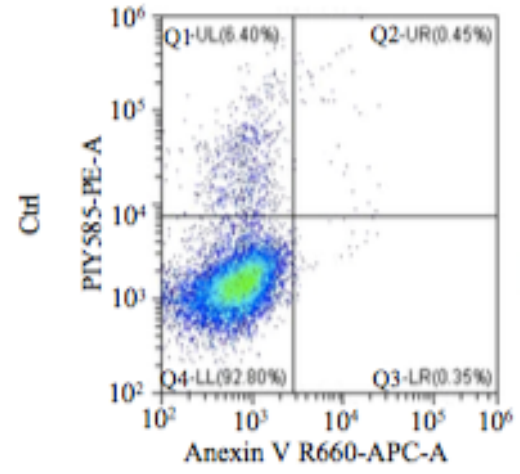

F

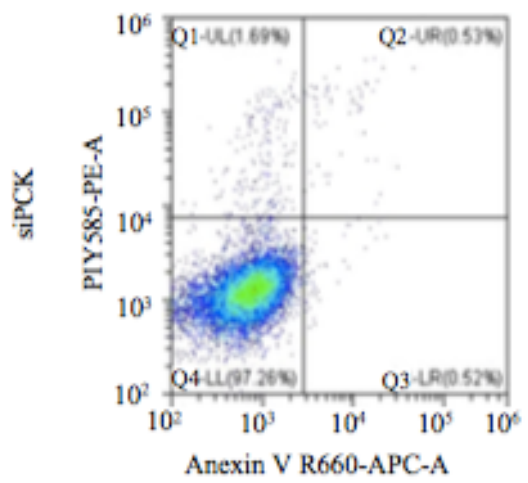

D

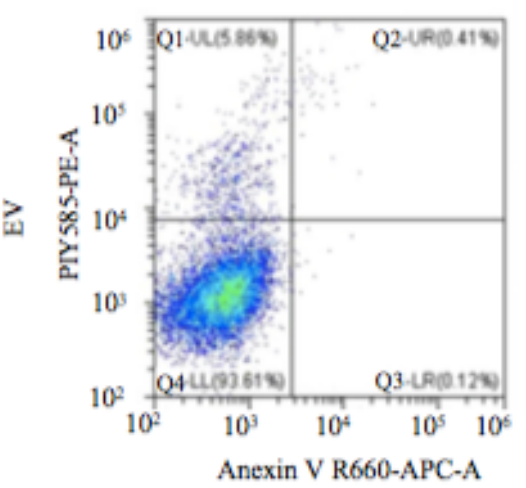

G

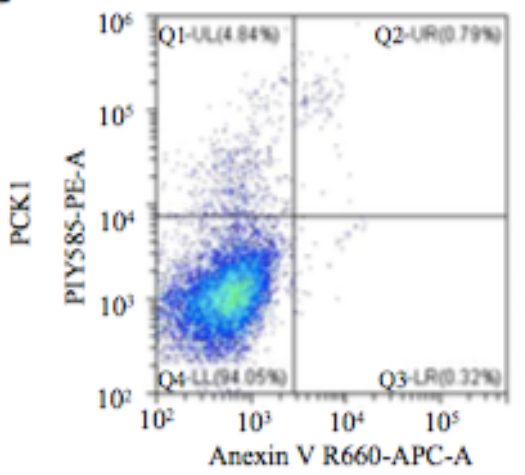

B

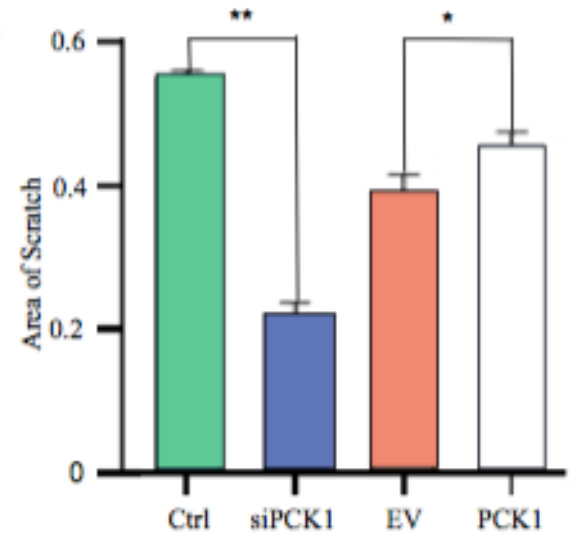

E

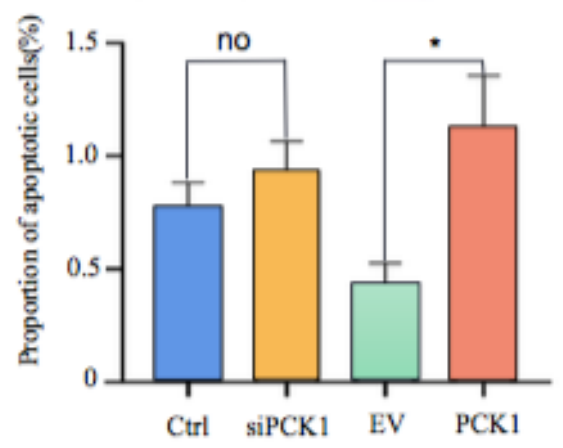

H

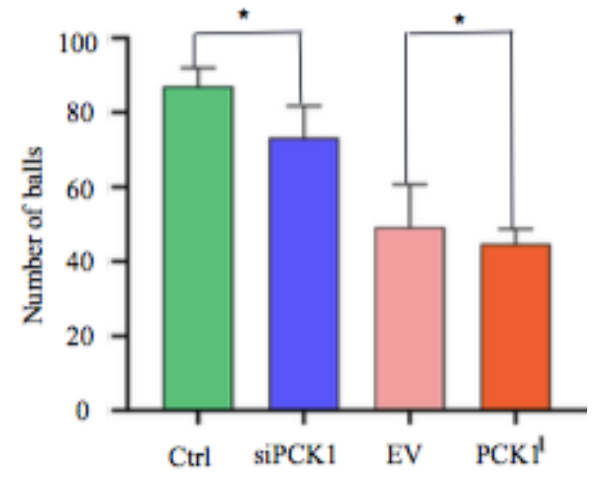

I

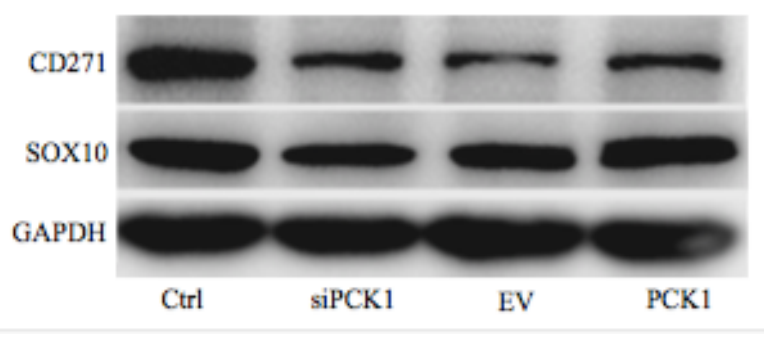

J

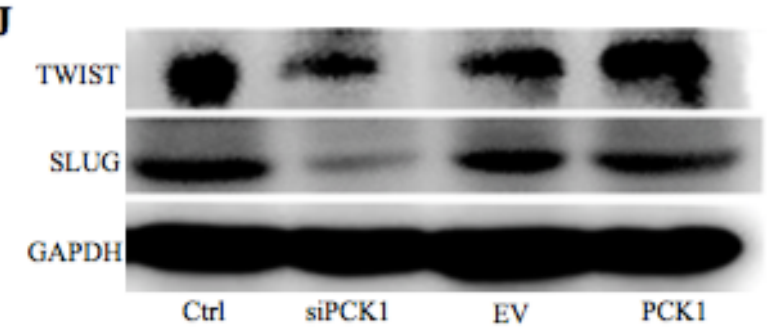




\section{Figure 4}

Functions of PCK1 in melanoma proliferation, migration, apoptosis, stemness, and EMT. A. Scratch assay. B. Results of the scratch assay: the $y$-axis is the area of change before and after scratching, and the proliferation area of SIPCK1 cells was smaller than that of Ctrl cells, and the proliferation area of PCK1 cells was higher than that of EV $(P<0.05)$. C.Flow cytometry of apoptosis: the y-axis represents the PIY585 staining and the x-axis represents the Annexin V staining. the Q2 + Q3 is the proportion of apoptotic cells. The percentage of apoptotic cells was $0.9 \%$ in the control group . D.Flow cytometry of apoptosis: the percentage of apoptotic cells was $0.53 \%$ in the EV group. E. Flow cytometry to assess apoptosis: there was no significant difference in the distribution of apoptotic cells.F. Flow cytometry to assess apoptosis: the percentage of apoptotic cells in the siPCK1 group was $1.05 \%$. G. Flow cytometry to assess apoptosis: the percentage of overexpression of the PCK 1 group was $1.11 \%$. H. Results of the sphere formation assay: the $y$-axis indicated the number of cell spheres with a diameter larger than 50 $\mu \mathrm{m}$ under a field of view, which was significantly lower in the siPCK1 group than in the Ctrl group, and higher in the overexpression PCK1 group than in the EV group, $\mathrm{P}<0.05$. I. Stemness test. J. EMT test.

$\mathbf{A}$

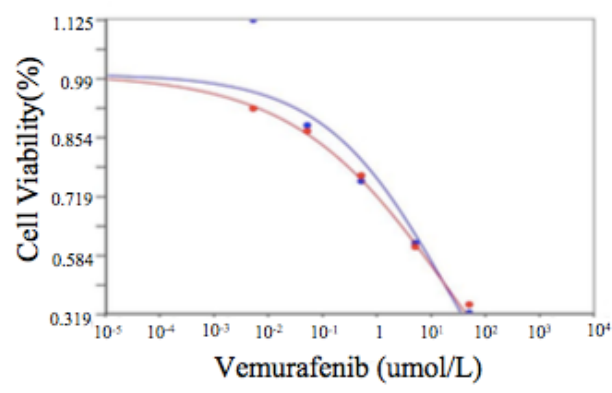

D

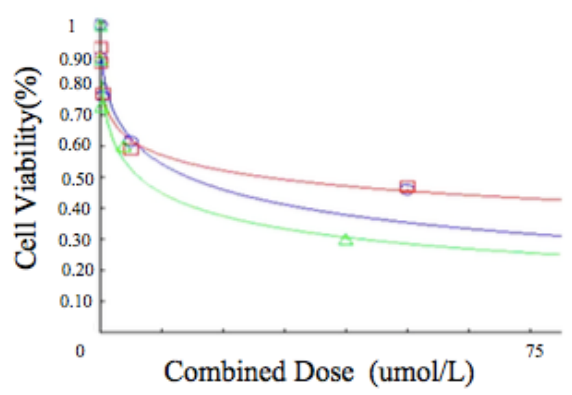

B

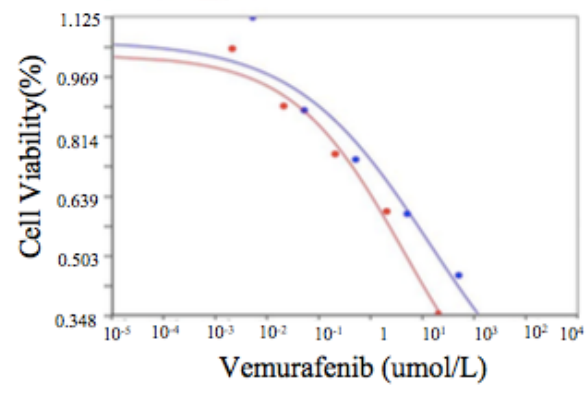

$\mathbf{E}$

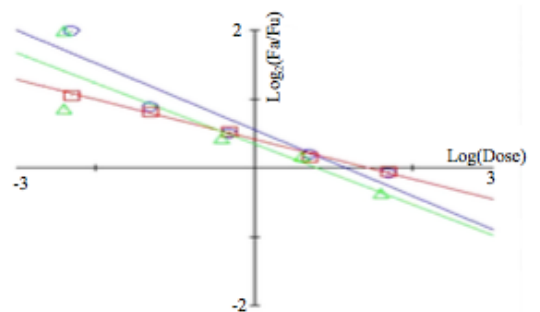

C

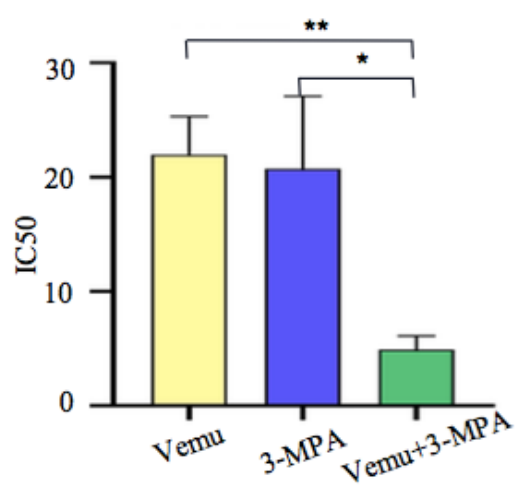

$\mathbf{F}$

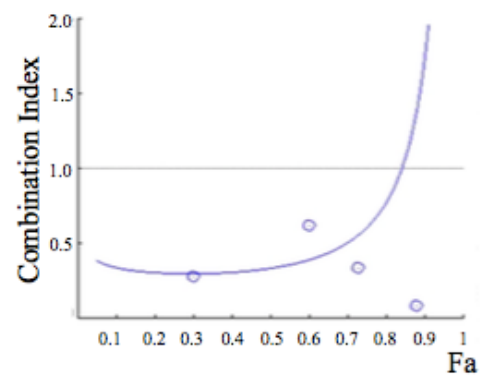

\section{Figure 5}

Synergism of PCK1 inhibitor 3-MPA in combination with vemurafenib in resistant cells. A. Cell viability curve: the blue dots and lines represent A2058R cells treated with vemurafenib, and the red dots and lines represent the A2058R cells treated with 3-MPA. B. Cell viability curve: the blue dots and lines represent A2058R cells treated with vemurafenib, and the red dots and lines represent the A2058R cells treated with a combination of 3-MPA and vemurafenib. C. IC50 statistical plot: the y-axis represents the IC50 value, the 
yellow color represents the A2058R cells treated with vemurafenib, the blue color represents the A2058R cells treated with 3-MPA, the green color represents a combination treatment with 3-MPA and vemurafenib. IC50 in A2058R cells treated with a combination of 3-MPA and vemurafenib, which was significantly lower than in A2058R cells treated with 3-MPA or vemurafenib alone, $P<0.05$. D. Cell viability curve based on the Chou-Talalay model: the blue triangles and lines represent the A2058R cells treated with 3-MPA, and the red and green colors represent those treated with vemurafenib, and with a combination of 3-MPA and vemurafenib, respectively. E. Median-Effect Plot: Fa and Fu represent the reacted and the unreacted parts in the reaction system, respectively; $\mathrm{Fa}+\mathrm{Fu}=1$. The $\mathrm{x}$-axis represents the drug dose. The combination index $(\mathrm{Cl})=0.3362<1$ showed that the two drugs played a synergistic role.

F. Combination index plot: The blue triangle and line represent the combination treatment with 3-MPA and vemurafenib. When $0.8<\mathrm{Fa}<0.9$, the combination index $(\mathrm{Cl})>1$, and the combined effect turned into antagonism.

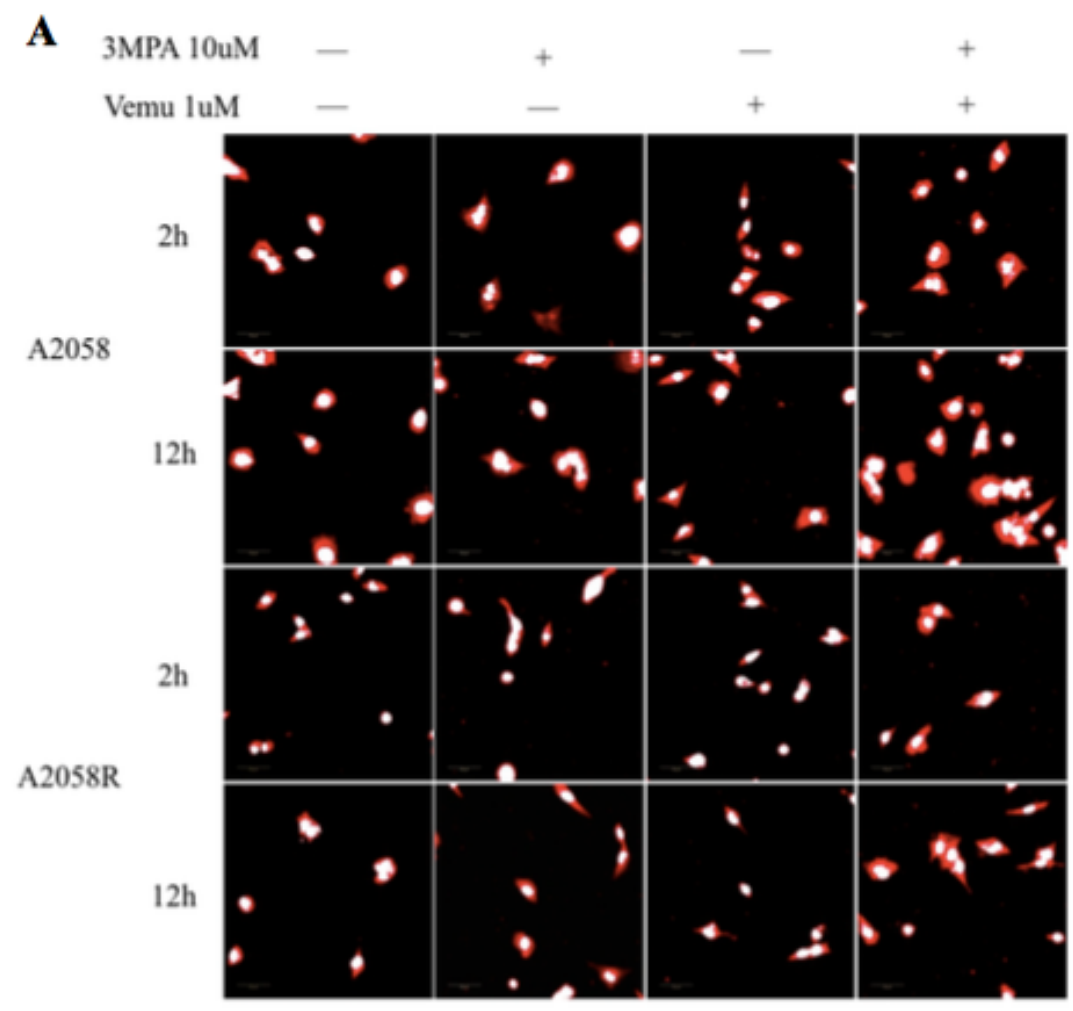

C

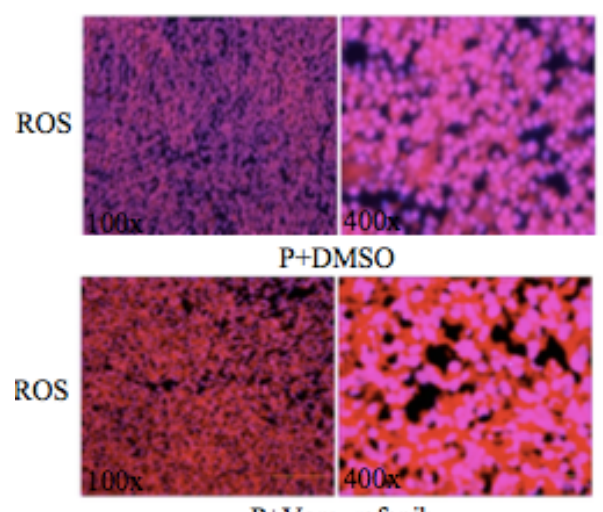

$\mathrm{P}+$ Vemurafenib

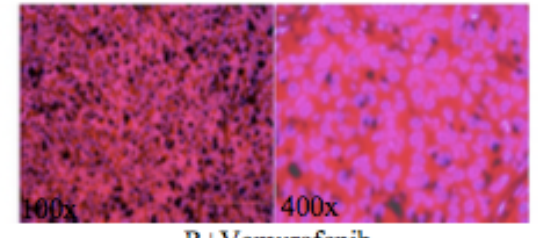

$\mathrm{R}+$ Vemurafenib

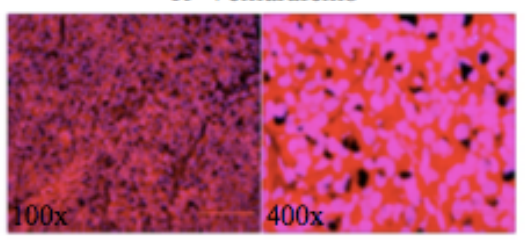

$\mathrm{R}+3-\mathrm{MPA}+$ Vemurafenib
B
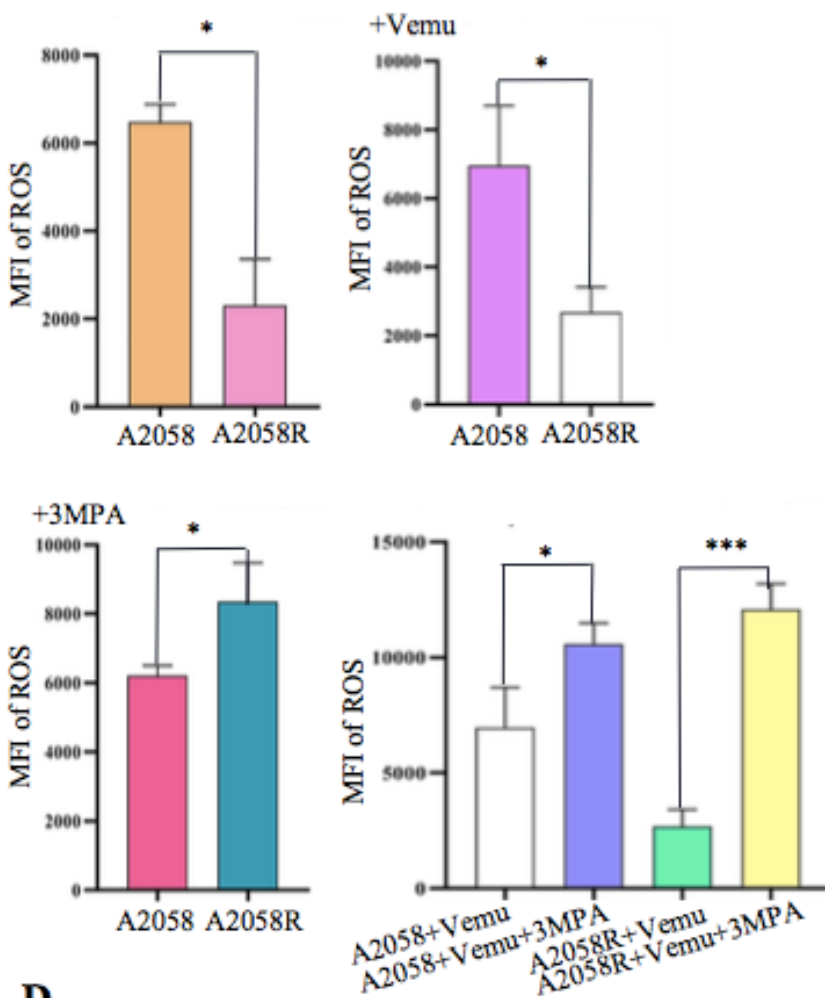

D

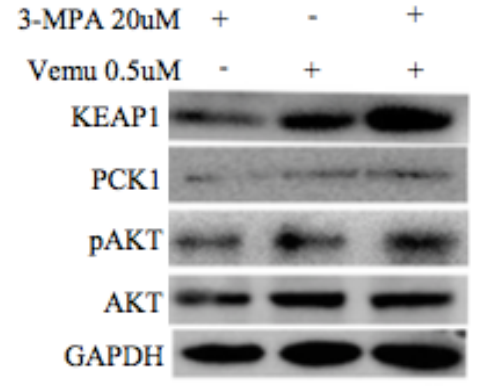

A2058

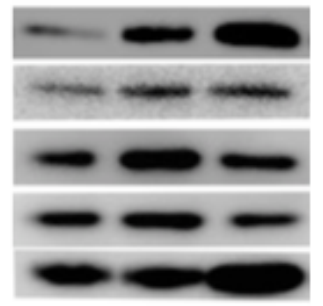

A2058R 


\section{Figure 6}

PCK1 led to drug resistance by reducing ROS accumulation. A. ROS imaging: The red area is the result of the post development of the ROS probe combined with intracytoplasmic ROS, and the white area represents the strongly refractile nuclear components. B. Result of ROS imaging: the y-axis represents the average light intensity of the probe, and the ROS content of A2058 cells was higher than that of A2058R cells. A2058 and A2058R cells showed increased ROS levels when vemurafenib was used alone, and A2058 cells showed higher ROS levels than A2058R cells $(P<0.05)$. After the addition of 3-MPA alone, the ROS content of A2058R cells was significantly higher than that of A2058 cells $(P<0.05)$. A2058 and A2058R cells showed increased ROS levels after the combination treatment with 3-MPA and vemurafenib, compared with the treatment with vemurafenib alone $(P<0.05)$. C. Detection of ROS in frozen section: the sliced tissues were derived from the subcutaneous tumorigenesis nude mice model, $\mathrm{P}$ represents the subcutaneous tumor formed after inoculation with melanoma A2058 cells, R represents the subcutaneous tumor formed after inoculation with drug-resistant A2058R cells, red represents the ROS, blue represents the nuclei (DAPI). ROS activity was higher within the tissues from $\mathrm{P}$ which received vemurafenib than from the $P$ which received DMSO and was lower within the tissues from $R$ which received vemurafenib; however, when 3-MPA and vemurafenib were combined, ROS activity was significantly higher within the tissues from $\mathrm{R}$ which received vemurafenib alone. D. Expression of KEAP1: in A2058 and A2058R cells, KEAP1 expression increased after the addition of 3-MPA. 
A

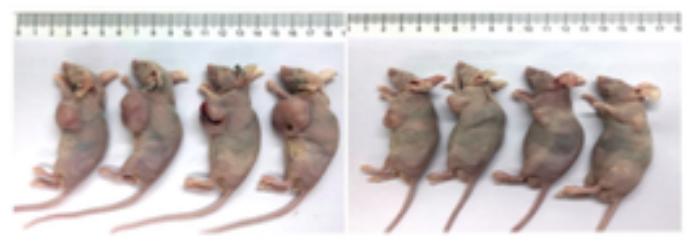

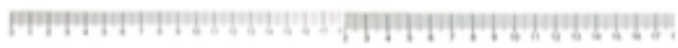

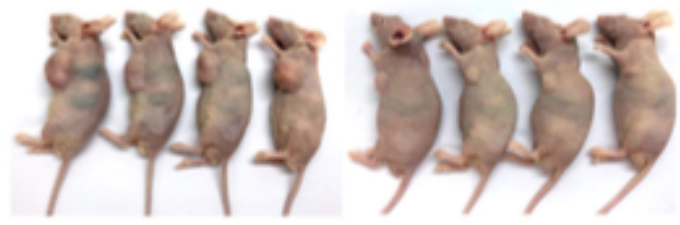

C

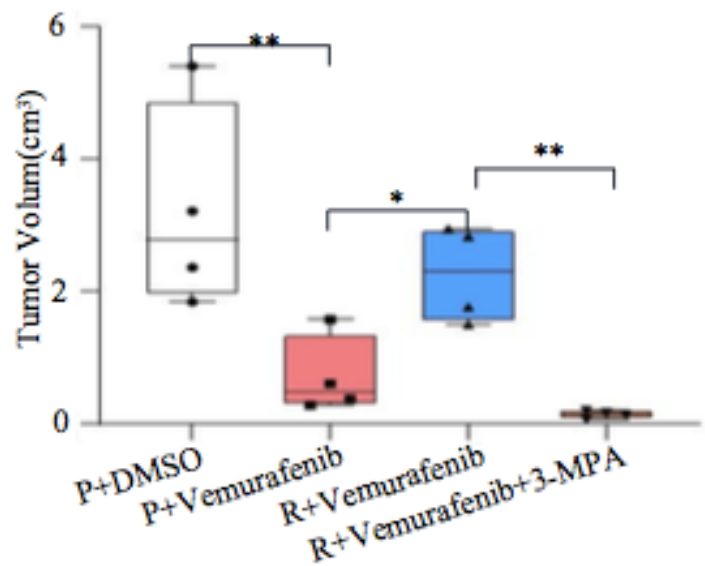

B

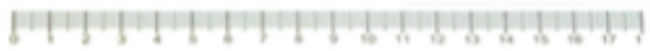

P + DMSO
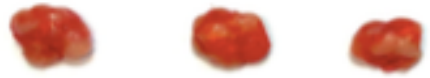

$\mathrm{P}+$ Vemu
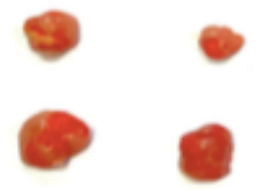

R+Vemu+3-MPA

D

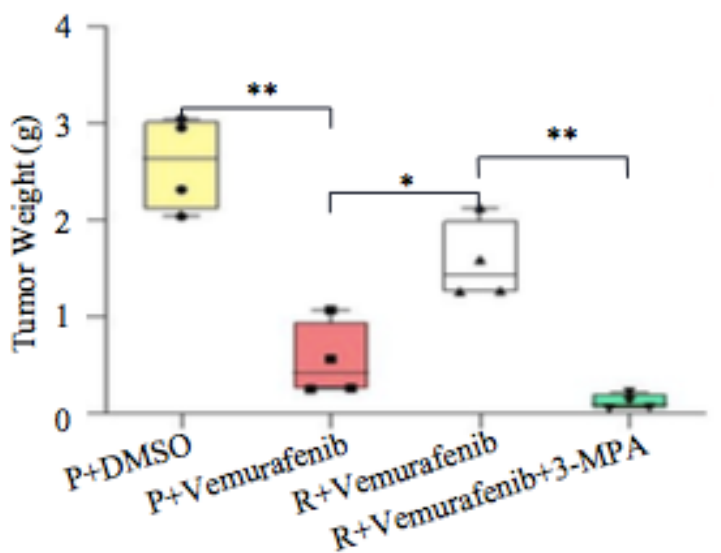

$\mathbf{E}$

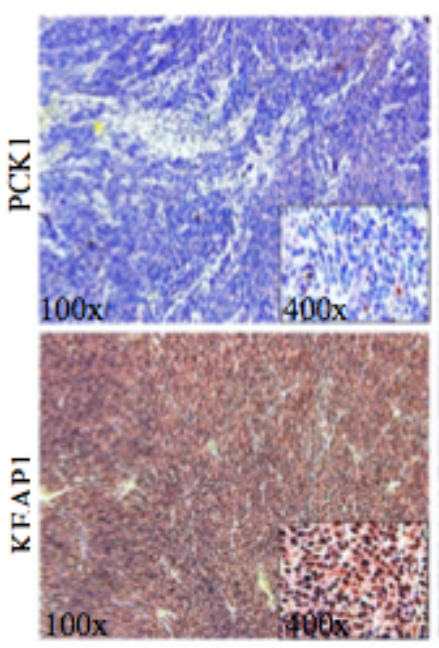

P+DMSO

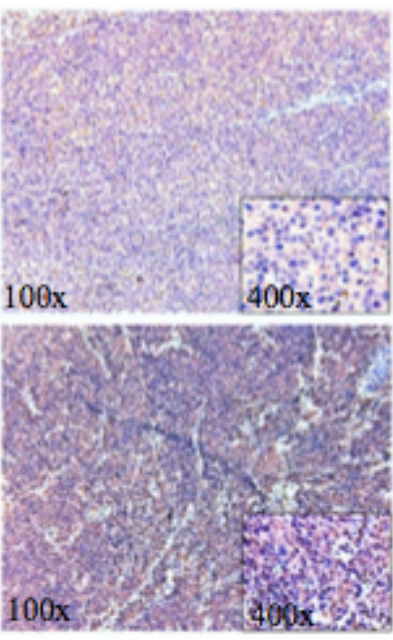

$\mathrm{P}+$ Vemurafenib
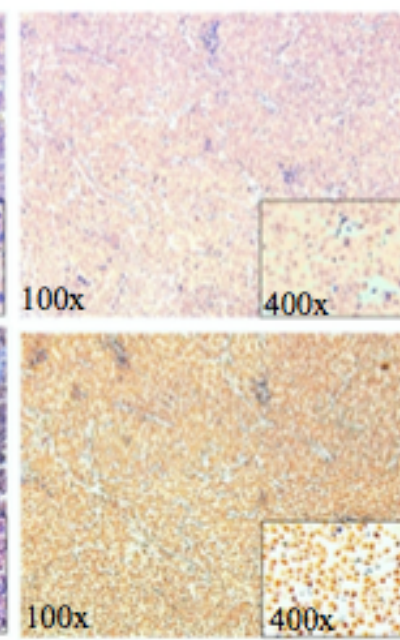

$\mathrm{R}+$ Vemurafenib

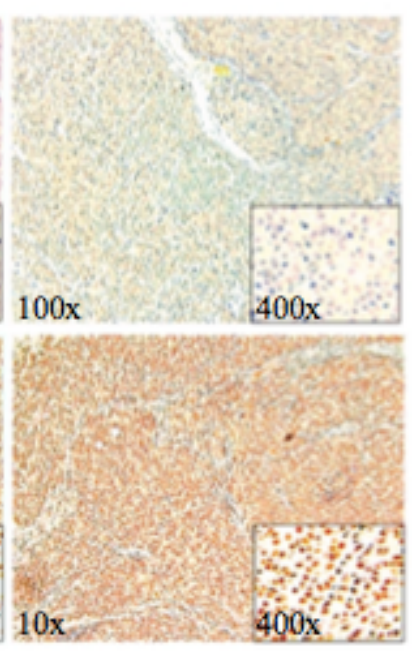

R+3-MPA+Vemurafenib

\section{Figure 7}

Verification of the chemoresistance mechanism of PCK1 and the benefit of combination therapy in vivo. A. Nude mice model of subcutaneous tumorigenesis. B. Subcutaneous tumor tissue. C. Distribution of the tumor volume: the tumor volume of the combination group was the lowest. D. Distribution of the tumor $w t$ : the tumor wt of the combination group was the lowest. E. Immunohistochemical results: the immunohistochemical staining of KEAP1 and PCK1 is shown in yellow; blue represents the nuclei (DAPI). 
In non-drug resistant tissues P, the expression of PCK1 increased and that of KEAP1 decreased after using vemurafenib. In R, the level of PCK1 was higher than that in P; the combination of 3-MPA and vemurafenib decreased the levels of PCK1 and increased the levels of KEAP1.

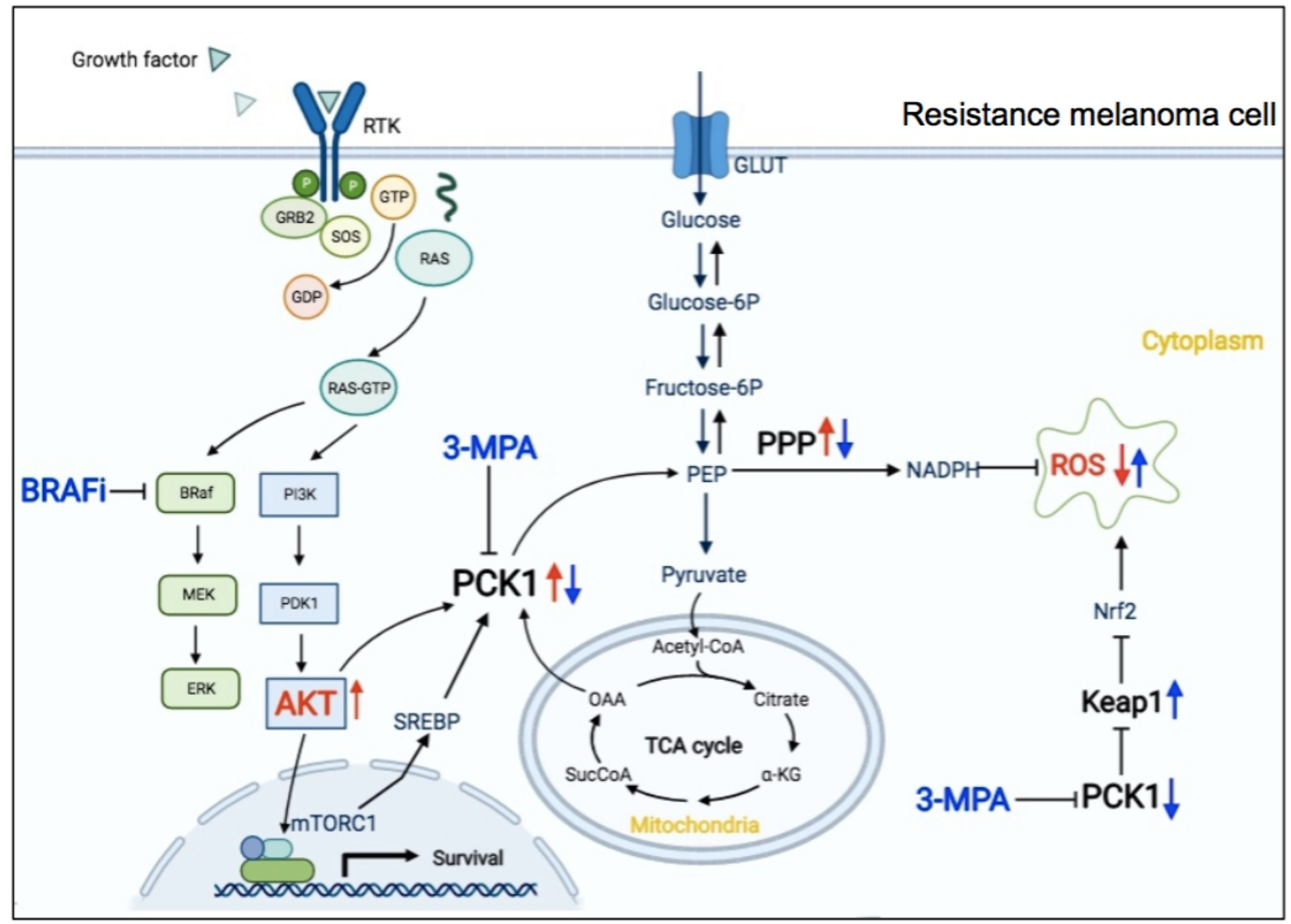

\section{Figure 8}

Mechanism of PCK1 and 3-MPA in resistant melanoma cells. In BRAFi-resistant melanoma cells with V600E mutation, AKT/PI3K signaling pathway was activated compensably, which promoted the synthesis of PCK1 and phosphorylated PCK1. On the one hand, it mediated the upregulation of the PPP metabolic pathway, synthesized a large amount of NADPH, reduced the intracellular ROS levels; on the other hand, it inhibited KEAP1, released the restriction of Nrf2, and activated the antioxidant effect of cells. All of these promoted the development of tumor drug resistance. When combined with 3-MPA, the synthesis of NADPH by the PPP decreased, and the antioxidant activity of cells was inhibited, which led to the accumulation of ROS and the oxidative attack of drug-resistant cells that were vulnerable to oxidative stress, thus exerting and strengthening the killing effect induced by vemurafenib.

\section{Supplementary Files}


This is a list of supplementary files associated with this preprint. Click to download.

- supplimentarytable1.xlsx 